\title{
Decision Support Technique Based on Spherical Fuzzy Yager Aggregation Operators and Their Application in Wind Power Plant Locations: A Case Study of Jhimpir, Pakistan
}

\author{
Ronnason Chinram $\mathbb{D}^{1},{ }^{1}$ Shahzaib Ashraf $\mathbb{D}^{2}{ }^{2}$ Saleem Abdullah, ${ }^{3}$ \\ and Pattarawan Petchkaew ${ }^{4}$ \\ ${ }^{1}$ Algebra and Applications Research Unit, Division of Computational Science, Faculty of Science, Prince of Songkla University, \\ Hat Yai, Songkhla 90110, Thailand \\ ${ }^{2}$ Department of Mathematics and Statistics, Bacha Khan University, Charsadda, Khyber Pakhtunkhwa, Pakistan \\ ${ }^{3}$ Department of Mathematics, Abdul Wali Khan University, Mardan, Khyber Pakhtunkhwa, Pakistan \\ ${ }^{4}$ Mathematics Program, Faculty of Science and Technology, Songkhla Rajabhat University, Songkhla, 90000, Thailand
}

Correspondence should be addressed to Shahzaib Ashraf; shahzaibashraf@bkuc.edu.pk

Received 27 September 2020; Revised 24 November 2020; Accepted 1 December 2020; Published 22 December 2020

Academic Editor: Tahir Mahmood

Copyright $\odot 2020$ Ronnason Chinram et al. This is an open access article distributed under the Creative Commons Attribution License, which permits unrestricted use, distribution, and reproduction in any medium, provided the original work is properly cited.

\begin{abstract}
The problem of energy crisis and environmental pollution has been mitigated by the generation and use of wind power; however, the choice of locations for wind power plants is a difficult task because the decision-making process includes political, socioeconomic, and environmental aspects. Thus, several adverse consequences have been created by the choice of suboptimal locations. The objective of this paper is to address the integrated qualitative and quantitative multicriteria decision-making framework for the selection of wind power plant locations. Spherical fuzzy sets are the latest extension of the ordinary fuzzy sets. The main characteristic of the spherical fuzzy sets is satisfying the condition that the squared sum of the positive, neutral, and negative grades must be at least zero and at most one. In this research, we establish novel operational laws based on the Yager $t$ norm and $t$-conorm under spherical fuzzy environments (SFE). Furthermore, based on these Yager operational laws, we develop list of novel aggregation operators under SFE. In addition, we design an algorithm to tackle the uncertainty to investigating the best wind power plant selection in four potential locations in Pakistan. A numerical example of wind power plant location problem is considered to show the supremacy and effectiveness of the proposed study. Also, a detailed comparison is constructed to evaluate the performance and validity of the established technique.
\end{abstract}

\section{Introduction}

One of the common and daily activity in humans' life is decision-making, aiming to choose the optimal alternative with respect to a list of attributes. Due to high capacity of decision-making to model the uncertainty of information, it has been widely studied and successfully applied to economics, management, and the other fields in recent years. Due to the uncertainty of decision information, utilizing fuzzy set theory to settle decision-making problem has become a hotspot in recent years. The concept of fuzzy set (FS) theory was firstly proposed by Zadeh [1], and, since then, the FSs have been widely used in many decision-making (DM) problems. FSs theory is a useful and appropriate approach to handle inaccurate and uncertain information in vague situations. Since the introduction of the FSs by Zadeh, they have been accepted and widespread in nearly all branches of science. Many extensions of ordinary FSs have been introduced by many researchers [2-7]. These extensions have been used frequently in the progress of DM problems in an uncertain environment. Some commonly used extensions of ordinary FSs will be explained in the following.

Intuitionistic FS is firstly established by Atanassov by adding the negative membership grades. Intuitionistic FS is 
the important generation of FS theory to tackle the uncertainty in complex real-life DM problems with more effective and efficient way. Many researchers contribute to intuitionistic FS theory; for example, Xu and Yager [8] introduced the geometric means-based aggregation operators (AOs) under intuitionistic FSs. Xu [9] established list of novel AOs to tackle the complex uncertainty under intuitionistic fuzzy settings. Wang and Liu [10] proposed the Einstein norm-based AOs using intuitionistic FSs. Yu and $\mathrm{Xu}$ [11] utilized prioritized concept to establish new AOs for intuitionistic FSs. Munezza et al. [12] presented the multicriteria DM system using cubic intuitionistic FS to tackle the uncertainty in location selection DM problem of small hydropower plant. Khan et al. [13] presented the novel DM approach under generalized intuitionistic fuzzy soft sets to tackle the incomplete information in daily life decision problems.

Although intuitionistic FS can deal with incomplete and uncertainty information, it cannot handle inconsistent information better in real situations. For example, in the work of Son [14], in the election of village director, the voting results can be divided into three categories: "vote for," "neutral voting," and "vote against." "Neutral voting" means that the voting paper is a white paper rejecting both agree and disagree for the candidate, but it still takes the vote. This example happened in reality, but intuitionistic FS could not handle it. In order to solve these problems, Cuong et al. $[15,16]$ proposed picture FS, which contains three aspects of information: yes, neutral, and no. It can deal with inconsistent information. Up to now, many outstanding contributions have been made in the research of picture FSs; for example, Wei [17] introduced some novel AOs for picture FS and discussed their applications in DM problems. Ashraf et al. [18] highlighted the deficiency in the existing operational laws and established novel improved AOs to tackle the uncertainty in complex real-life DM problems under picture fuzzy environment. Khan et al. [19] established the novel extension, generalized picture fuzzy soft sets, and discussed their DM applications. Khan et al. [20] established the novel AOs using logarithmic function and algebraic norm under picture fuzzy environment. Qiyas et al. [21] presented the linguistic information and algebraic norm-based novel AOs using picture FSs. Ashraf et al. [22] presented the cleaner production evaluation technique based on the cubic picture fuzzy AOs using distance information measures. Qiyas et al. [23] utilized linguistic variables to develop the list of AOs based on Dombi operational laws to tackle the DM problems of real word. Ashraf and Abdullah [24] introduced algebraic norm-based AOs under cubic picture FS and discussed their applications in decision problem.

Picture FS is an important generalization of FS theory, but, with the constant complexity of human knowledge modeling and theory development, picture FS will be invalid in some DM problems. Ashraf et al. [25, 26] introduced a new and more general concept spherical fuzzy set (spherical FS), which is an extension of FS by further slackening the condition that $0 \leq \mu^{2}(v)+\wp^{2}(v)+\partial^{2}(v) \leq 1$. We must also note that the acceptable spherical space provides more freedom for observers to express their belief in supporting membership. Therefore, spherical FSs express more extensive fuzzy information, while spherical FSs are more maneuverable and more appropriate for dealing with uncertainties information. However, spherical FSs have been successfully applied in some fields, especially in decisionmaking fields.

As aggregation operators have a strong role in DM problems, several researchers have made quite valuable contributions to introduce aggregation operators for spherical FS. Spherical aggregation operators based on algebraic norms [26] deal with uncertainty and inaccurate information in DM problems. Spherical FS representations of spherical fuzzy norms [27] are introduced under SF information. SF Dombi aggregation operators based on Dombi norm are introduced in [28]. SF logarithmic aggregation operators based on entropy are proposed in [29]. Linguistic SF aggregation operators are presented in [30] for SF information to tackle the uncertainty in DMP. GRA methodology based on spherical linguistic fuzzy Choquet integral is proposed [31] for SF information. Cosine similarity measures are presented in [32] to discuss the application in DMP. Application of SF distance measures is discussed in [33] to determine the child development influence environmental factors using SF information. In [34], the TOPSIS approach based on SF rough set was proposed and its application in DMP was discussed. Gü ndoğdu and Kahraman [35] established the TOPSIS methodology under spherical FSs and also proposed its applications. Ashraf and Abdullah [36] presented the emergency decision-making technique of coronavirus using the spherical FSs. Ashraf et al. [37] introduced the symmetric sum-based AOs under spherical FSs to tackle the uncertainty in daily life DM problems. Gundogdu and Kahraman [38] established the generalized methodology based on WASPAS under spherical FSs. Shishavan et al. [39] established the list of similarity measures to tackle the uncertainty in the form of spherical fuzzy environment. Gündoğdu and Kahraman [40] presented the new AHP technique to tackle the uncertainty in renewable energy and in [41] discussed the spherical fuzzy QFD technique to tackle the uncertainty in robot technology development problems.

It is evident that the abovementioned AOs are focused on the algebraic, Einstein, Dombi, and Hamacher norms under spherical FSs for the implementation of the combination process. Algebraic, Einstein, Dombi, and Hamacher product and sum are not only fundamental spherical FS operations that describe the union and the intersection of any two spherical FSs. A general union and intersection under SF information can be developed from a generalized norm; that is, instances of deferent-norms families may be used to execute the respective intersections and unions under spherical fuzzy environment. The Yager product and sum are good replacement of the algebraic, Einstein, Dombi, and Hamacher product for an intersection and union and are capable of delivering smooth estimates of the algebraic product and sum. However, there seems to be little work in the literature on aggregation approaches that use the Yager operations on FS theory to aggregate the fuzzy numbers. Akram and Shahzadi [42] introduced the q-rung orthopair 
FS-based Yager AOs to tackle the DM problems. Akram et al. [43] presented the Yager norm-based AOs under complex Pythagorean FSs and discussed their application in DM problems. Shahzadi et al. [44] presented the DM approach based on Yager operational laws under Pythagorean information. Garg et al. [45] presented the DM problem of COVID-19 testing facility using Fermatean FS and Yager norm information.

From the above analysis, we note that, in many practical applications, various aggregation operators have been put forward and implemented. Although in practical problems many existing AOs are not able to address such specific cases, in some circumstances, many of these may result in unreasonable or counterintuitive results. Certain new regulations built without a simple function may have a complicated description. But generalized aggregation operators for SFSs continue to be an open subject that attracts the attention of many researchers. Therefore, in this article, our aim is to present some novel spherical fuzzy Yager operational laws based AOs to tackle the uncertainty in real-world DM problems with more effective and efficient way. The contribution and originality of this study are summarized as follows:

(i) Novel ranking methodology and Yager norm-based novel operational laws for spherical fuzzy sets are proposed

(ii) The new spherical fuzzy Yager averaging/geometric aggregation operators are proposed to aggregate the uncertainties in the form of spherical fuzzy environment

(iii) Decision-making algorithm is proposed to tackle the real-world DM problems

(iv) A real-life numerical application about wind power plant location selection problem in Pakistan is discussed to show the applicability of the proposed technique

The rest of this article shall be organized as set out below. Section 2 provides basic information concerning spherical FSs. Section 3 describes the Yager operations of spherical FSs. Section 4 proposes a new way to rank the spherical fuzzy number with more consistency. Section 5 , presented as the cornerstone of this work, proposes novel spherical fuzzy Yager AOs based on the Yager norm, together with the associated proof of its properties. Section 6 introduces the novel methodology for interacting with the ambiguity in DM problems in order to pick the best alternative according to the list of attributes. Section 7 provides a numerical application about wind power plant location selection problem used to illustrate the designed MAGDM method and a comparative analysis with some existing frameworks of MAGDM is discussed in Section 8. The article is concluded in Section 9.

\section{Preliminaries}

Let us briefly recall in this segment the rudiments of FSs and spherical FSs. For the following review, these definitions will be included here.
Definition 1 (see [1]). A fuzzy set (FS) $F$ in a universe set $U$ is an object having the form

$$
F=\{\langle v, \mu(v)\rangle \mid v \in U\}
$$

where $\mu(v) \in[0,1]$ is represented by the positive membership grade.

Definition 2 (see [46]). An intuitionistic FS $F_{s}$ in a universe set $U$ is an object having the form

$$
F_{s}=\{\langle v, \mu(v), \partial(v)\rangle \mid v \in U\},
$$

where $\mu(v) \in[0,1]$ and $\partial(v) \in[0,1]$ are positive and negative membership grades, respectively. In addition, $0 \leq \mu(v)+\partial(v) \leq 1, \forall v \in U$.

Definition 3 (see [47]). A Pythagorean FS $F_{s}$ in a universe set $U$ is an object having the form

$$
F_{s}=\{\langle v, \mu(v), \partial(v)\rangle \mid v \in U\},
$$

where $\mu(v) \in[0,1]$ and $\partial(v) \in[0,1]$ are positive and negative membership grades, respectively. In addition, $0 \leq \mu^{2}(v)+\partial^{2}(v) \leq 1, \forall v \in U$.

Definition 4 (see [15]). A picture FS $F_{s}$ in a universe set $U$ is an object having the form

$$
F_{s}=\{\langle v, \mu(v), \wp(v), \partial(v)\rangle \mid v \in U\},
$$

where $\mu(v) \in[0,1], \wp(v) \in[0,1]$, and $\partial(v) \in[0,1]$ are positive, neutral, and negative membership grades, respectively. In addition, $0 \leq \mu(v)+\wp(v)+\partial(v) \leq 1, \forall v \in U$.

Definition 5 (see $[25,26])$. A spherical FS $F_{s}$ in a universe set $U$ is an object having the form

$$
F_{s}=\{\langle v, \mu(v), \wp(v), \partial(v)\rangle \mid v \in U\},
$$

where $\mu(v) \in[0,1], \wp(v) \in[0,1]$, and $\partial(v) \in[0,1]$ are positive, neutral, and negative membership grades, respectively. In addition, $0 \leq \mu^{2}(v)+\wp^{2}(v)+\partial^{2}(v) \leq 1, \forall v \in U$.

In what follows, we signify by SFS $(U)$ the family of all spherical FSs. We shall signify the spherical fuzzy number (SFN) with the triplet $F_{\mathrm{ts}}=(\mu(v), \wp(v), \partial(v))$ for simplicity.

Definition 6 (see [25]). Suppose that, for any $F_{\mathrm{ts}_{(1)}}, F_{\mathrm{ts}_{(2)}} \in \mathrm{SFS}(U)$.

(1) $F_{\mathrm{ts}_{(1)}} \subseteq F_{\mathrm{ts}_{(2)}}$ if and only if $\mu_{1} \leq \mu_{2}, \wp_{1} \leq \wp_{2}$ and $\partial_{1} \geq \partial_{2}$. Clearly, $F_{\mathrm{ts}_{(1)}}=F_{\mathrm{ts}_{(2)}}$ if $F_{\mathrm{ts}_{(1)}} \subseteq F_{\mathrm{ts}_{(2)}}$ and $F_{\mathrm{ts}_{(2)}} \subseteq F_{\mathrm{ts}_{(1)}}$.

(2) $F_{\mathrm{ts}_{(1)}} \cap F_{\mathrm{ts}_{(2)}}=\left\{\min \left(\mu_{1}, \mu_{2}\right), \min \left(\wp_{1}, \wp_{2}\right), \max \left(\partial_{1}, \partial_{2}\right)\right\}$.

(3) $F_{\mathrm{ts}_{(1)}} \cup F_{\mathrm{ts}_{(2)}}=\left\{\max \left(\mu_{1}, \mu_{2}\right), \min \left(\wp_{1}, \wp_{2}\right)\right.$, $\left.\min \left(\partial_{1}, \partial_{2}\right)\right\}$.

(4) $F_{\mathrm{ts}_{(1)}}^{c}=\left\{\partial_{1}, \wp_{1}, \mu_{1}\right\}$.

Definition 7 (see [25]). Let $F_{\mathrm{ts}_{(1)}}, F_{\mathrm{ts}_{(2)}} \in \mathrm{SFS}(U)$ with $\varrho>0$. The operating laws are as follows:
(1) $F_{\mathrm{ts}_{(1)}} \otimes F_{\mathrm{ts}_{(2)}}=\left\{\mu_{1} \mu_{2}, \wp_{1} \wp_{2}, \sqrt{\partial_{1}^{2}+\partial_{2}^{2}-\partial_{1}^{2} \partial_{2}^{2}}\right\}$ 
(2) $F_{\mathrm{ts}_{(1)}} \oplus F_{\mathrm{ts}_{(2)}}=\left\{\sqrt{\mu_{1}^{2}+\mu_{2}^{2}-\mu_{1}^{2} \mu_{2}^{2}}, \wp_{1} \wp_{2}, \partial_{1} \partial_{2}\right\}$

(3) $F_{\mathrm{ts}_{(1)}}^{\varrho}=\left\{\mu_{1}^{\varrho}, \wp_{1}^{\varrho}, \sqrt{1-\left(1-\partial_{1}^{2}\right)^{\varrho}}\right\}$

(4) $\varrho \cdot F_{\mathrm{ts}_{(1)}}=\left\{\sqrt{1-\left(1-\mu_{1}^{2}\right)^{\varrho}}, \wp_{1}^{\varrho}, \partial_{1}^{\varrho}\right\}$

Definition 8 (see $[25,48])$. Let $F_{\mathrm{ts}_{(1)}}=\left(\mu_{1}, \wp_{1}, \partial_{1}\right)$ and $F_{\mathrm{ts}_{(2)}}=\left(\mu_{2}, \wp_{2}, \partial_{2}\right) \in \mathrm{SFS}(U) . \quad \widehat{S}_{c}\left(F_{\mathrm{ts}_{(1)}}\right)=\mu_{1}^{2}-\partial_{1}^{2} \quad$ and $\widehat{S} c\left(F_{\mathrm{ts}}\right)=\mu_{2}^{2}-\partial_{2}^{2}$ are the score values of SFNs. Also $\widehat{A} c\left(F_{\mathrm{ts}_{(1)}}\right)=\mu_{1}^{2}+\wp_{1}^{2}+\partial_{1}^{2}$ and $\widehat{A} c\left(F_{\mathrm{ts}_{(2)}}\right)=\mu_{2}^{2}+\wp_{2}^{2}+\partial_{2}^{2}$ are the accuracy values of SFNs. We have the following: (a) $\widehat{S} c\left(F_{\mathrm{ts}_{(1)}}\right)<\widehat{S} c\left(F_{\mathrm{ts}_{(2)}}\right) \Longrightarrow F_{\mathrm{ts}_{(1)}}<F_{\mathrm{ts}_{(2)}}$

(b) $\widehat{S} c\left(F_{\mathrm{ts}_{(1)}}\right)=\widehat{S} c\left(F_{\mathrm{ts}_{(2)}}\right), \widehat{A} c\left(F_{\mathrm{ts}_{(1)}}\right)<\widehat{A} c\left(F_{\mathrm{ts}_{(2)}}\right) \Longrightarrow$ $F_{\mathrm{ts}_{(1)}}<F_{\mathrm{ts}_{(2)}}$

(c) $\widehat{S} c\left(F_{\mathrm{ts}_{(1)}}\right)=\widehat{S} c\left(F_{\mathrm{ts}_{(2)}}\right), \widehat{A} c\left(F_{\mathrm{ts}_{(1)}}\right)=\widehat{A} c\left(F_{\mathrm{ts}_{(2)}}\right) \Longrightarrow$ $F_{\mathrm{ts}_{(1)}}=F_{\mathrm{ts}_{(2)}}$

Definition 9 (see [26]). Let $F_{g}=\left(\mu_{g}(v), \wp_{g}(v), \partial_{g}(v)\right) \in$ SFN $(U)(g=1,2,3, \ldots, n)$. Then, the weighted averaging AOs for SFN $(U)$ are described as

$$
\begin{aligned}
\operatorname{SFWA}\left(F_{1}, F_{2}, \ldots, F_{n}\right) & =\ell_{1} F_{1} \oplus \ell_{2} F_{2} \oplus \cdots \oplus \ell_{n} F_{n} \\
& =\sum_{g=1}^{n} \ell_{g} F_{g} \\
& =\left\{\sqrt{\left.1-\prod_{g=1}^{n}\left(1-\mu_{g}^{2}\right)^{\ell_{g}}, \prod_{g=1}^{n}\left(\wp_{g}\right)^{\ell_{g}}, \prod_{g=1}^{n}\left(\partial_{g}\right)^{\ell_{g}}\right\},}\right.
\end{aligned}
$$

where the weights $\left(\ell_{1}, \ell_{2}, \ldots, \ell_{g}\right)$ of $F_{g}$ have $\ell_{g} \geq 0$ and $\sum_{g=1}^{n} \ell_{g}=1$.
Definition 10 (see [26]). Let $F_{g}=\left(\mu_{g}(v), \wp_{g}(v)\right.$, $\left.\partial_{g}(v)\right) \in \operatorname{SFN}(U)(g=1,2,3, \ldots, n)$. Then, the weighted geometric AOs for SFN $(U)$ are described as

$$
\begin{aligned}
\operatorname{SFWG}\left(F_{1}, F_{2}, \ldots, F_{n}\right) & =F_{1}^{\ell_{1}} \otimes F_{2}^{\ell_{2}} \otimes \cdots \otimes F_{n}^{\ell_{n}} \\
& =\prod_{g=1}^{n}\left(F_{g}\right)^{\ell_{g}} \\
& =\left\{\prod_{g=1}^{n}\left(\mu_{g}\right)^{\ell_{g}}, \prod_{g=1}^{n}\left(\wp_{g}\right)^{\ell_{g}}, \sqrt{1-\prod_{g=1}^{n}\left(1-\partial_{g}^{2}\right)^{\ell_{g}}}\right\},
\end{aligned}
$$

where the weights $\left(\ell_{1}, \ell_{2}, \ldots, \ell_{g}\right)$ of $F_{g}$ have $\ell_{g} \geq 0$ and $\sum_{g=1}^{n} \ell_{g}=1$.

\section{New Operating Laws for Spherical FS}

Aggregation operators (AOs) play an essential part in combining data into one form and in tackling MCGDM problems. Aggregation facilitates the establishment of a number of choices in a system or a collection of objects that have come together or have been brought together. In recent years, AOs based on FSs and their different hybrid compositions have provided a great deal of attention and have become interesting because they can quickly execute functional areas of various regions. In this section, we propose the Yager norms-based novel operational laws for spherical FNs.

Definition 11 (see [49]). Suppose that, for any real numbers $l$ and $m$, Yager's norms have the forms
(1) $\check{T}(l, m)=1-\min \left(1,\left((1-l)^{\delta}+(1-m)^{\delta}\right)^{1 / \delta}\right)$;

(2) $\widehat{S}(l, m)=\min \left(1,\left(l^{\delta}-m^{\delta}\right)^{1 / \delta}\right), \delta \in(0, \infty)$.

Definition 12. Let $F_{\mathrm{ts}_{(1)}}, F_{\mathrm{ts}_{(2)}} \in \mathrm{SFS}(U)$ with $\varrho, \delta>0$. The Yager operating laws (YOLs) are $F_{\mathrm{ts}}=(\mu(v), \wp(v), \partial(v))$ and are described as follows:

(1) $F_{\mathrm{ts}_{(1)}} \otimes F_{\mathrm{ts}_{(2)}}$

$$
\begin{aligned}
& =\left\{\sqrt{1-\min \left(1,\left(\left(1-\mu_{1}^{2}\right)^{\delta}+\left(1-\mu_{2}^{2}\right)^{\delta}\right)^{1 / \delta}\right)},\right. \\
& \sqrt{1-\min \left(1,\left(\left(1-\wp_{1}^{2}\right)^{\delta}+\left(1-\wp_{2}^{2}\right)^{\delta}\right)^{1 / \delta}\right)}, \\
& \left.\sqrt{\min \left(1,\left(\partial_{1}^{2 \delta}+\partial_{2}^{2 \delta}\right)^{1 / \delta}\right)}\right\}
\end{aligned}
$$

(2) $F_{\mathrm{ts}_{(1)}} \oplus F_{\mathrm{ts}_{(2)}}=\left\{\sqrt{\min \left(1,\left(\mu_{1}^{2 \delta}+\mu_{2}^{2 \delta}\right)^{1 / \delta}\right)}\right.$,

$$
\begin{aligned}
& \sqrt{1-\min \left(1,\left(\left(1-\wp_{1}^{2}\right)^{\delta}+\left(1-\wp_{2}^{2}\right)^{\delta}\right)^{1 / \delta}\right)}, \\
& \left.\sqrt{1-\min \left(1,\left(\left(1-\partial_{1}^{2}\right)^{\delta}+\left(1-\partial_{2}^{2}\right)^{\delta}\right)^{1 / \delta}\right)}\right\}
\end{aligned}
$$


Journal of Mathematics

5

(3)

$$
\begin{aligned}
& F_{\mathrm{ts}(1)}^{\varrho}=\left\{\sqrt{1-\min \left(1,\left(\varrho\left(1-\mu_{1}^{2}\right)^{\delta}\right)^{1 / \delta}\right)}\right. \\
& \left.\sqrt{1-\min \left(1,\left(\varrho\left(1-\wp_{1}^{2}\right)^{\delta}\right)^{1 / \delta}\right)}, \sqrt{\min \left(1,\left(\varrho \partial_{1}^{2 \delta}\right)^{1 / \delta}\right)}\right\}
\end{aligned}
$$

(4)

$$
\begin{aligned}
& \varrho \cdot F_{\mathrm{ts}_{(1)}}=\left\{\sqrt{\min \left(1,\left(\varrho \mu_{1}^{2 \delta}\right)^{1 / \delta}\right)},\right. \\
& \sqrt{1-\min \left(1,\left(\varrho\left(1-\wp_{1}^{2}\right)^{\delta}\right)^{1 / \delta}\right)} \\
& \left.\sqrt{1-\min \left(1,\left(\varrho\left(1-\partial_{1}^{2}\right)^{\delta}\right)^{1 / \delta}\right)}\right\}
\end{aligned}
$$

Theorem 1. Let $F_{t s_{(1)}}, F_{t s_{(2)}} \in S F S(U)$ with $\varrho_{1}, \varrho_{2}>0$. Then,
(1) $F_{\mathrm{ts}_{(1)}} \oplus F_{\mathrm{ts}_{(2)}}=F_{\mathrm{ts}_{(2)}} \oplus F_{\mathrm{ts}_{(1)}}$

(2) $F_{\mathrm{ts}_{(1)}} \otimes F_{\mathrm{ts}_{(2)}}=F_{\mathrm{ts}_{(2)}} \otimes F_{\mathrm{ts}_{(1)}}$

(3) $\varrho\left(F_{\mathrm{ts}_{(1)}} \oplus F_{\mathrm{ts}_{(2)}}\right)=\varrho F_{\mathrm{ts}_{(1)}} \oplus \varrho F_{\mathrm{ts}_{(2)}}$

(4) $\left(\varrho_{1}+\varrho_{2}\right) F_{\mathrm{ts}_{(1)}}=\varrho_{1} F_{\mathrm{ts}_{(1)}} \oplus \varrho_{2} F_{\mathrm{ts}_{(1)}}$

(5) $\left(F_{\mathrm{ts}_{(1)}} \otimes F_{\mathrm{ts}_{(2)}}\right)^{\varrho}=F_{\mathrm{ts}_{(1)}}^{\varrho} \otimes F_{\mathrm{ts}_{(2)}}^{\varrho}$

(6) $F_{\mathrm{ts}_{(1)}}^{\mathrm{Q}_{1}} \otimes F_{\mathrm{ts}_{(1)}}^{\mathrm{Q}_{2}}=F_{\mathrm{ts}_{(1)}}^{\left(\varrho_{1}+\varrho_{2}\right.}$

Proof. For any $F_{\mathrm{ts}_{(1)}}, F_{\mathrm{ts}_{(2)}} \in \mathrm{SFS}(U)$ with $\varrho_{1}, \varrho_{2}>0$, we have

$$
\begin{aligned}
& F_{\mathrm{ts}_{(1)}} \oplus F_{\mathrm{ts}_{(2)}}=\left\{\sqrt{\min \left(1,\left(\mu_{1}^{2 \delta}+\mu_{2}^{2 \delta}\right)^{1 / \delta}\right)}, \sqrt{1-\min \left(1,\left(\left(1-\wp_{1}^{2}\right)^{\delta}+\left(1-\wp_{2}^{2}\right)^{\delta}\right)^{1 / \delta}\right)}, \sqrt{1-\min \left(1,\left(\left(1-\partial_{1}^{2}\right)^{\delta}+\left(1-\partial_{2}^{2}\right)^{\delta}\right)^{1 / \delta}\right)}\right\} \\
& =\left\{\sqrt{\min \left(1,\left(\mu_{2}^{2 \delta}+\mu_{1}^{2 \delta}\right)^{1 / \delta}\right)}, \sqrt{1-\min \left(1,\left(\left(1-\wp_{2}^{2}\right)^{\delta}+\left(1-\wp_{1}^{2}\right)^{\delta}\right)^{1 / \delta}\right)}, \sqrt{1-\min \left(1,\left(\left(1-\partial_{2}^{2}\right)^{\delta}+\left(1-\partial_{1}^{2}\right)^{\delta}\right)^{1 / \delta}\right)}\right\} \\
& =F_{\mathrm{ts}_{(2)}} \oplus F_{\mathrm{ts}_{(1)}} \\
& F_{\mathrm{ts}_{(1)}} \otimes F_{\mathrm{ts}_{(2)}}=\left\{\sqrt{1-\min \left(1,\left(\left(1-\mu_{1}^{2}\right)^{\delta}+\left(1-\mu_{2}^{2}\right)^{\delta}\right)^{1 / \delta}\right)}, \sqrt{1-\min \left(1,\left(\left(1-\wp_{1}^{2}\right)^{\delta}+\left(1-\wp_{2}^{2}\right)^{\delta}\right)^{1 / \delta}\right)}, \sqrt{\min \left(1,\left(\partial_{1}^{2 \delta}+\partial_{2}^{2 \delta}\right)^{1 / \delta}\right)}\right\} \\
& =\left\{\sqrt{1-\min \left(1,\left(\left(1-\mu_{2}^{2}\right)^{\delta}+\left(1-\mu_{1}^{2}\right)^{\delta}\right)^{1 / \delta}\right)}, \sqrt{1-\min \left(1,\left(\left(1-\wp_{2}^{2}\right)^{\delta}+\left(1-\wp_{1}^{2}\right)^{\delta}\right)^{1 / \delta}\right)}, \sqrt{\min \left(1,\left(\partial_{2}^{2 \delta}+\partial_{1}^{2 \delta}\right)^{1 / \delta}\right)}\right\} \\
& =F_{\mathrm{ts}_{(2)}} \otimes F_{\mathrm{ts}_{(1)}} \text {, } \\
& \varrho\left(F_{\mathrm{ts}_{(1)}} \oplus F_{\mathrm{ts}_{(2)}}\right)=\varrho \cdot\left\{\sqrt{\min \left(1,\left(\mu_{1}^{2 \delta}+\mu_{2}^{2 \delta}\right)^{1 / \delta}\right)}, \sqrt{1-\min \left(1,\left(\left(1-\wp_{1}^{2}\right)^{\delta}+\left(1-\wp_{2}^{2}\right)^{\delta}\right)^{1 / \delta}\right)}, \sqrt{1-\min \left(1,\left(\left(1-\partial_{1}^{2}\right)^{\delta}+\left(1-\partial_{2}^{2}\right)^{\delta}\right)^{1 / \delta}\right)}\right\} \\
& =\left\{\sqrt{\min \left(1,\left(\varrho \mu_{1}^{2 \delta}+\varrho \mu_{2}^{2 \delta}\right)^{1 / \delta}\right)}, \sqrt{1-\min \left(1,\left(\varrho\left(1-\wp_{1}^{2}\right)^{\delta}+\varrho\left(1-\wp_{2}^{2}\right)^{\delta}\right)^{1 / \delta}\right)}, \sqrt{1-\min \left(1,\left(\varrho\left(1-\partial_{1}^{2}\right)^{\delta}+\varrho\left(1-\partial_{2}^{2}\right)^{\delta}\right)^{1 / \delta}\right)}\right\} \\
& \varrho F_{\mathrm{ts}_{(1)}} \oplus \varrho F_{\mathrm{ts}_{(2)}}=\left\{\sqrt{\min \left(1,\left(\varrho \mu_{1}^{2 \delta}\right)^{1 / \delta}\right)}, \sqrt{1-\min \left(1,\left(\varrho\left(1-\wp_{1}^{2}\right)^{\delta}\right)^{1 / \delta}\right)}, \sqrt{1-\min \left(1,\left(\varrho\left(1-\partial_{1}^{2}\right)^{\delta}\right)^{1 / \delta}\right)}\right\} \\
& \oplus\left\{\sqrt{\min \left(1,\left(\varrho \mu_{2}^{2 \delta}\right)^{1 / \delta}\right)}, \sqrt{1-\min \left(1,\left(\varrho\left(1-\gamma_{2}^{2}\right)^{\delta}\right)^{1 / \delta}\right)}, \sqrt{1-\min \left(1,\left(\varrho\left(1-\partial_{2}^{2}\right)^{\delta}\right)^{1 / \delta}\right)}\right\} \\
& =\left\{\sqrt{\min \left(1,\left(\varrho \mu_{1}^{2 \delta}+\varrho \mu_{2}^{2 \delta}\right)^{1 / \delta}\right)}, \sqrt{1-\min \left(1,\left(\varrho\left(1-\wp_{1}^{2}\right)^{\delta}+\varrho\left(1-\wp_{2}^{2}\right)^{\delta}\right)^{1 / \delta}\right)}, \sqrt{1-\min \left(1,\left(\varrho\left(1-\partial_{1}^{2}\right)^{\delta}+\varrho\left(1-\partial_{2}^{2}\right)^{\delta}\right)^{1 / \delta}\right)}\right\}, \\
& \Rightarrow \varrho\left(F_{\mathrm{ts}_{(1)}} \oplus F_{\mathrm{ts}_{(2)}}\right)=\varrho F_{\mathrm{ts}_{(1)}} \oplus \varrho F_{\mathrm{ts}_{(2)}} \text {. } \\
& \varrho_{1} F_{\mathrm{ts}_{(1)}} \oplus \varrho_{2} F_{\mathrm{ts}_{(1)}}=\left\{\sqrt{\min \left(1,\left(\varrho_{1} \mu_{1}^{2 \delta}\right)^{1 / \delta}\right)}, \sqrt{1-\min \left(1,\left(\varrho_{1}\left(1-\wp_{1}^{2}\right)^{\delta}\right)^{1 / \delta}\right)}, \sqrt{1-\min \left(1,\left(\varrho_{1}\left(1-\partial_{1}^{2}\right)^{\delta}\right)^{1 / \delta}\right)}\right\} \\
& \oplus\left\{\sqrt{\min \left(1,\left(\varrho_{2} \mu_{2}^{2 \delta}\right)^{1 / \delta}\right)}, \sqrt{1-\min \left(1,\left(\varrho_{2}\left(1-\wp_{2}^{2}\right)^{\delta}\right)^{1 / \delta}\right)}, \sqrt{1-\min \left(1,\left(\varrho_{2}\left(1-\partial_{2}^{2}\right)^{\delta}\right)^{1 / \delta}\right)}\right\} \\
& =\left\{\sqrt{\min \left(1,\left(\left(\varrho_{1}+\varrho_{2}\right) \mu_{1}^{2 \delta}\right)^{1 / \delta}\right)}, \sqrt{1-\min \left(1,\left(\left(\varrho_{1}+\varrho_{2}\right)\left(1-\wp_{1}^{2}\right)^{\delta}\right)^{1 / \delta}\right)}, \sqrt{1-\min \left(1,\left(\left(\varrho_{1}+\varrho_{2}\right)\left(1-\partial_{1}^{2}\right)^{\delta}\right)^{1 / \delta}\right)}\right\} \\
& =\left(\varrho_{1}+\varrho_{2}\right) F_{\mathrm{ts}_{(1)}} \text {. } \\
& \varrho_{1} F_{\mathrm{ts}_{(1)}} \oplus \varrho_{2} F_{\mathrm{ts}_{(1)}}=\left\{\sqrt{\min \left(1,\left(\varrho_{1} \mu_{1}^{2 \delta}\right)^{1 / \delta}\right)}, \sqrt{1-\min \left(1,\left(\varrho_{1}\left(1-\wp_{1}^{2}\right)^{\delta}\right)^{1 / \delta}\right)}, \sqrt{1-\min \left(1,\left(\varrho_{1}\left(1-\partial_{1}^{2}\right)^{\delta}\right)^{1 / \delta}\right)}\right\} \\
& \oplus\left\{\sqrt{\min \left(1,\left(\varrho_{2} \mu_{2}^{2 \delta}\right)^{1 / \delta}\right)}, \sqrt{1-\min \left(1,\left(\varrho_{2}\left(1-\wp_{2}^{2}\right)^{\delta}\right)^{1 / \delta}\right)}, \sqrt{1-\min \left(1,\left(\varrho_{2}\left(1-\partial_{2}^{2}\right)^{\delta}\right)^{1 / \delta}\right)}\right\} \\
& =\left\{\sqrt{\min \left(1,\left(\left(\varrho_{1}+\varrho_{2}\right) \mu_{1}^{2 \delta}\right)^{1 / \delta}\right)}, \sqrt{1-\min \left(1,\left(\left(\varrho_{1}+\varrho_{2}\right)\left(1-\wp_{1}^{2}\right)^{\delta}\right)^{1 / \delta}\right)}, \sqrt{1-\min \left(1,\left(\left(\varrho_{1}+\varrho_{2}\right)\left(1-\partial_{1}^{2}\right)^{\delta}\right)^{1 / \delta}\right)}\right\} \\
& =\left(\varrho_{1}+\varrho_{2}\right) F_{\mathrm{ts}_{(1)}}
\end{aligned}
$$


Proofs of (5) and (6) are similar as above.

\section{A New Way to Rank SFNs}

Here, we will construct a new procedure for the ranking of SFNs in the present section. This new framework is being used to rank SFNs and to choose the best alternative. From Definition 8, we know that Mahmood et al. [48] have given us a ranking method of SFNs. Therefore, this framework is sensitive to a slight change in the SFNs, as shown by the example below.

Example 1. Suppose the following: $F_{\mathrm{ts}}=(0.7,0.5,0.66)$, $F_{\mathrm{ts}_{(\alpha 2)}}=(0.7,0.5,0.6599), \quad F_{\mathrm{ts}_{(\beta 1)}}=(0.8,0.3,0.77), \quad$ and $F_{\mathrm{ts}_{(\beta 2)}}=(0.8,0.3,0.7699) \in \mathrm{SFS}(U)$. Then, by Definition 8 , we have

$$
\begin{aligned}
& \widehat{S} c\left(F_{t s_{(\alpha 1)}}\right)=0.055504, \widehat{S} c\left(F_{t s_{(\alpha 2)}}\right)=0.055634 \\
& \widehat{S} c\left(F_{t s_{(\beta 1)}}\right)=0.055467, \widehat{S} c\left(F_{t s_{(\beta 2)}}\right)=0.055644 .
\end{aligned}
$$

Since $\quad \widehat{S}_{c}\left(F_{\mathrm{ts}_{(\alpha 1)}}\right)>\widehat{S}_{c}\left(F_{\mathrm{ts}_{(\beta 1)}}\right) \quad$ and $\quad \widehat{S}_{c}\left(F_{\mathrm{ts}_{(\alpha 2)}}\right)<\widehat{S}_{c}\left(F_{\mathrm{ts}_{(\beta 2)}}\right)$, $F_{\mathrm{ts}_{(\alpha 1)}}>F_{\mathrm{ts}_{(\beta 1)}}$ and $F_{\mathrm{ts}_{(\alpha 2)}}<F_{\mathrm{ts}_{(\beta 2)}}$.
The findings in the above example demonstrate that the SFNs ranking, which is assessed on the basis of Definition 8, would absolutely change, even if the SFNs slightly change. A new ranking way is proposed in this article in order to overcome the shortcomings of the ranking framework based on Definition 8. The novel ranking framework is proposed in the following definition.

Definition 13. Let $F_{\mathrm{ts}_{(1)}}=\left(\mu_{1}, \wp_{1}, \partial_{1}\right) \in \mathrm{SFS}(U)$. Then, the score value $\widehat{S} c$ is described as

$$
\widehat{S} c\left(F_{\mathrm{ts}_{(1)}}\right)=\frac{\left(\left(1-\partial_{1}\right)^{2}+1-\partial_{1}^{2}\right)^{1 / 2}}{\left(\left(1-\mu_{1}\right)^{2}+1-\mu_{1}^{2}\right)^{1 / 2}+\left(\left(1-\partial_{1}\right)^{2}+1-\partial_{1}^{2}\right)^{1 / 2}}
$$

where $\widehat{S}_{c}\left(F_{\mathrm{ts}_{(1)}}\right) \in[0,1]$.

Theorem 2. Let $F_{t s_{(1)}}=\left(\mu_{1}, \wp_{1}, \partial_{1}\right) \in S F S(U)$. If $\mu_{1}=\partial_{1}$, then $\widehat{S}_{c}\left(F_{t s_{(1)}}\right)=1 / 2$; if $\mu_{1}>\partial_{1}$, then $\widehat{S}_{c}\left(F_{t s_{(1)}}\right)>1 / 2$, and if $\mu_{1}<\partial_{1}$, then $\widehat{S}_{c}\left(F_{t s_{(1)}}\right)<1 / 2$.

Proof. Let $F_{\mathrm{ts}_{(1)}}=\left(\mu_{1}, \wp_{1}, \partial_{1}\right) \in \mathrm{SFS}(U)$. If $\mu_{1}=\partial_{1}$, then

$$
\widehat{S} c\left(F_{\mathrm{ts}_{(1)}}\right)=\frac{\left(\left(1-\partial_{1}\right)^{2}+1-\partial_{1}^{2}\right)^{1 / 2}}{\left(\left(1-\mu_{1}\right)^{2}+1-\mu_{1}^{2}\right)^{1 / 2}+\left(\left(1-\partial_{1}\right)^{2}+1-\partial_{1}^{2}\right)^{1 / 2}}=\frac{\left(\left(1-\partial_{1}\right)^{2}+1-\partial_{1}^{2}\right)^{1 / 2}}{\left(\left(1-\partial_{1}\right)^{2}+1-\partial_{1}^{2}\right)^{1 / 2}+\left(\left(1-\partial_{1}\right)^{2}+1-\partial_{1}^{2}\right)^{1 / 2}}=\frac{1}{2} .
$$

If $\mu_{1}>\partial_{1}$, then

$$
\widehat{S} c\left(F_{\mathrm{ts}_{(1)}}\right)=\frac{\left(\left(1-\partial_{1}\right)^{2}+1-\partial_{1}^{2}\right)^{1 / 2}}{\left(\left(1-\mu_{1}\right)^{2}+1-\mu_{1}^{2}\right)^{1 / 2}+\left(\left(1-\partial_{1}\right)^{2}+1-\partial_{1}^{2}\right)^{1 / 2}}>\frac{\left(\left(1-\partial_{1}\right)^{2}+1-\partial_{1}^{2}\right)^{1 / 2}}{\left(\left(1-\partial_{1}\right)^{2}+1-\partial_{1}^{2}\right)^{1 / 2}+\left(\left(1-\partial_{1}\right)^{2}+1-\partial_{1}^{2}\right)^{1 / 2}}=\frac{1}{2} .
$$

If $\mu_{1}<\partial_{1}$, then

$$
\widehat{S} c\left(F_{\mathrm{ts}_{(1)}}\right)=\frac{\left(\left(1-\partial_{1}\right)^{2}+1-\partial_{1}^{2}\right)^{1 / 2}}{\left(\left(1-\mu_{1}\right)^{2}+1-\mu_{1}^{2}\right)^{1 / 2}+\left(\left(1-\partial_{1}\right)^{2}+1-\partial_{1}^{2}\right)^{1 / 2}}<\frac{\left(\left(1-\partial_{1}\right)^{2}+1-\partial_{1}^{2}\right)^{1 / 2}}{\left(\left(1-\partial_{1}\right)^{2}+1-\partial_{1}^{2}\right)^{1 / 2}+\left(\left(1-\partial_{1}\right)^{2}+1-\partial_{1}^{2}\right)^{1 / 2}}=\frac{1}{2} .
$$

The proof is completed.

Theorem 3. Let $F_{t s_{(1)}}=\left(\mu_{1}, \wp_{1}, \partial_{1}\right)$ and $F_{t s_{(2)}}=\left(\mu_{2}\right.$, $\left.\wp_{2}, \partial_{2}\right) \in S F S(U)$. If $\mu_{1}=\mu_{2}$ and $\partial_{1}<\partial_{2}$, then
$\widehat{S}_{c}\left(F_{t s_{(1)}}\right)>\widehat{S}_{c}\left(F_{t s_{(2)}}\right)$. Otherwise, if $\partial_{1}=\partial_{2}$ and $\mu_{1}>\mu_{2}$, then $\widehat{S}_{c}\left(F_{t s_{(1)}}\right)>\widehat{S}_{c}\left(F_{t s_{(2)}}\right)$.

Proof. Let $J=\widehat{S}_{c}\left(F_{\operatorname{ts}_{(1)}}\right) / \widehat{S}_{c}\left(F_{\operatorname{ts}_{(2)}}\right)$. According to Definition 13 , we have 


$$
\begin{aligned}
J= & \frac{\left(\left(1-\partial_{1}\right)^{2}+1-\partial_{1}^{2}\right)^{1 / 2} /\left(\left(\left(1-\mu_{1}\right)^{2}+1-\mu_{1}^{2}\right)^{1 / 2}+\left(\left(1-\partial_{1}\right)^{2}+1-\partial_{1}^{2}\right)^{1 / 2}\right)}{\left(\left(1-\partial_{2}\right)^{2}+1-\partial_{2}^{2}\right)^{1 / 2} /\left(\left(\left(1-\mu_{2}\right)^{2}+1-\mu_{2}^{2}\right)^{1 / 2}+\left(\left(1-\partial_{2}\right)^{2}+1-\partial_{2}^{2}\right)^{1 / 2}\right)} \\
= & \frac{\left(\left(1-\partial_{1}\right)^{2}+1-\partial_{1}^{2}\right)^{1 / 2}\left[\left(\left(1-\mu_{2}\right)^{2}+1-\mu_{2}^{2}\right)^{1 / 2}+\left(\left(1-\partial_{2}\right)^{2}+1-\partial_{2}^{2}\right)^{1 / 2}\right]}{\left(\left(1-\partial_{2}\right)^{2}+1-\partial_{2}^{2}\right)^{1 / 2}\left[\left(\left(1-\mu_{1}\right)^{2}+1-\mu_{1}^{2}\right)^{1 / 2}+\left(\left(1-\partial_{1}\right)^{2}+1-\partial_{1}^{2}\right)^{1 / 2}\right]} \\
= & \frac{\left[\left(\left(1-\partial_{1}\right)^{2}+1-\partial_{1}^{2}\right)^{1 / 2} \cdot\left(\left(1-\mu_{2}\right)^{2}+1-\mu_{2}^{2}\right)^{1 / 2}\right]+\left[\left(\left(1-\partial_{1}\right)^{2}+1-\partial_{1}^{2}\right)^{1 / 2} \cdot\left(\left(1-\partial_{2}\right)^{2}+1-\partial_{2}^{2}\right)^{1 / 2}\right]}{\left[\left(\left(1-\partial_{2}\right)^{2}+1-\partial_{2}^{2}\right)^{1 / 2} \cdot\left(\left(1-\mu_{1}\right)^{2}+1-\mu_{1}^{2}\right)^{1 / 2}\right]+\left[\left(\left(1-\partial_{2}\right)^{2}+1-\partial_{2}^{2}\right)^{1 / 2} \cdot\left(\left(1-\partial_{1}\right)^{2}+1-\partial_{1}^{2}\right)^{1 / 2}\right]} .
\end{aligned}
$$

Let $P=\left[\left(\left(1-\partial_{1}\right)^{2}+1-\partial_{1}^{2}\right)^{1 / 2} \cdot\left(\left(1-\partial_{2}\right)^{2}+1-\partial_{2}^{2}\right)^{1 / 2}\right]$; then we have

$$
J=\frac{P+\left[\left(\left(1-\partial_{1}\right)^{2}+1-\partial_{1}^{2}\right)^{1 / 2} \cdot\left(\left(1-\mu_{2}\right)^{2}+1-\mu_{2}^{2}\right)^{1 / 2}\right]}{P+\left[\left(\left(1-\partial_{2}\right)^{2}+1-\partial_{2}^{2}\right)^{1 / 2} \cdot\left(\left(1-\mu_{1}\right)^{2}+1-\mu_{1}^{2}\right)^{1 / 2}\right]} .
$$

If $\mu_{1}=\mu_{2}$ and $\partial_{1}<\partial_{2}$, then we have

$$
\begin{gathered}
J=\frac{P+\left[\left(\left(1-\partial_{1}\right)^{2}+1-\partial_{1}^{2}\right)^{1 / 2} \cdot\left(\left(1-\mu_{2}\right)^{2}+1-\mu_{2}^{2}\right)^{1 / 2}\right]}{P+\left[\left(\left(1-\partial_{2}\right)^{2}+1-\partial_{2}^{2}\right)^{1 / 2} \cdot\left(\left(1-\mu_{1}\right)^{2}+1-\mu_{1}^{2}\right)^{1 / 2}\right]} \\
>\frac{P+\left[\left(\left(1-\partial_{2}\right)^{2}+1-\partial_{2}^{2}\right)^{1 / 2} \cdot\left(\left(1-\mu_{2}\right)^{2}+1-\mu_{2}^{2}\right)^{1 / 2}\right]}{P+\left[\left(\left(1-\partial_{2}\right)^{2}+1-\partial_{2}^{2}\right)^{1 / 2} \cdot\left(\left(1-\mu_{2}\right)^{2}+1-\mu_{2}^{2}\right)^{1 / 2}\right]}
\end{gathered}
$$$$
=1 \text {. }
$$

Thus, $J=\left(\widehat{S} c\left(F_{\mathrm{ts}_{(1)}}\right) / \widehat{S} c\left(F_{\mathrm{ts}_{(2)}}\right)\right)>1 \Rightarrow \widehat{S} c\left(F_{\mathrm{ts}_{(1)}}\right)>\widehat{S} c\left(F_{\mathrm{ts}_{(2)}}\right)$. If $\partial_{1}=\partial_{2}$ and $\mu_{1}>\mu_{2}$, then we have

$$
\begin{gathered}
J=\frac{P+\left[\left(\left(1-\partial_{1}\right)^{2}+1-\partial_{1}^{2}\right)^{1 / 2} \cdot\left(\left(1-\mu_{2}\right)^{2}+1-\mu_{2}^{2}\right)^{1 / 2}\right]}{P+\left[\left(\left(1-\partial_{2}\right)^{2}+1-\partial_{2}^{2}\right)^{1 / 2} \cdot\left(\left(1-\mu_{1}\right)^{2}+1-\mu_{1}^{2}\right)^{1 / 2}\right]} \\
>\frac{P+\left[\left(\left(1-\partial_{2}\right)^{2}+1-\partial_{2}^{2}\right)^{1 / 2} \cdot\left(\left(1-\mu_{2}\right)^{2}+1-\mu_{2}^{2}\right)^{1 / 2}\right]}{P+\left[\left(\left(1-\partial_{2}\right)^{2}+1-\partial_{2}^{2}\right)^{1 / 2} \cdot\left(\left(1-\mu_{2}\right)^{2}+1-\mu_{2}^{2}\right)^{1 / 2}\right]}
\end{gathered}
$$

$$
=1 \text {. }
$$

Thus, $J=\left(\widehat{S}_{c}\left(F_{\mathrm{ts}_{(1)}}\right) / \widehat{S}_{c}\left(F_{\mathrm{ts}_{(2)}}\right)\right)>1 \Rightarrow \widehat{S}_{c}\left(F_{\mathrm{ts}_{(1)}}\right)>\widehat{S}_{c}\left(F_{\mathrm{ts}_{(2)}}\right)$. The proof is completed.
However, it is also observed that, in certain situations, the score function that we have described cannot distinguish SFNs. For example, consider the following: $F_{\mathrm{ts}_{(1)}}=$ $(0.6,0.5,0.6)$ and $F_{\mathrm{ts}_{(2)}}=(0.7,0.5,0.7) \in \mathrm{SFS}(U)$. It is not difficult to get that $\widehat{S} c\left(F_{\mathrm{ts}_{(1)}}\right)=\widehat{S} c\left(F_{\mathrm{ts}_{(2)}}\right)=0.5$ according to Definition 13. To equate SFNs in these situations, the accuracy function is established and a novel framework for ordering SFNs is proposed.

Definition 14. Let $F_{\mathrm{ts}_{(1)}}=\left(\mu_{1}, \wp_{1}, \partial_{1}\right)$ and $F_{\mathrm{ts}_{(2)}}=\left(\mu_{2}, \wp_{2}, \partial_{2}\right) \epsilon$ SFS $(U) . \widehat{S}_{c}\left(F_{\mathrm{ts}_{(1)}}\right)$ and $\widehat{S} c\left(F_{\mathrm{ts}_{(2)}}\right)$ are the score values of $F_{\mathrm{ts}_{(1)}}$ and $F_{\mathrm{ts}_{(2)}}$, and $\widehat{A} c\left(F_{\mathrm{ts}_{(1)}}\right)$ and $\widehat{A} c\left(F_{\mathrm{ts}_{(2)}}\right)$ are the accuracy values of $F_{\mathrm{ts}_{(1)}}$ and $F_{\mathrm{ts}_{(2)}}$, respectively.

(1) If $\widehat{S}_{c}\left(F_{\mathrm{ts}_{(1)}}\right)<\widehat{S}_{c}\left(F_{\mathrm{ts}_{(2)}}\right)$, then $F_{\mathrm{ts}_{(1)}}<F_{\mathrm{ts}_{(2)}}$

(2) If $\widehat{S}_{c}\left(F_{\mathrm{ts}_{(1)}}\right)=\widehat{S}_{c}\left(F_{\mathrm{ts}_{(2)}}\right)$, then
(a) if $\widehat{A} c\left(F_{\mathrm{ts}_{(1)}}\right)<\widehat{A} c\left(F_{\mathrm{ts}_{(2)}}\right)$, then $F_{\mathrm{ts}_{(1)}}<F_{\mathrm{ts}_{(2)}}$
(b) $\widehat{A} c\left(F_{\mathrm{ts}_{(1)}}\right)=\widehat{A} c\left(F_{\mathrm{ts}_{(2)}}\right)$, then $F_{\mathrm{ts}_{(1)}}=F_{\mathrm{ts}_{(2)}}$.

Example 2. Consider the following: $F_{\mathrm{ts}_{(\alpha 1)}}=(0.7,0.5,0.66)$, $F_{\mathrm{ts}_{(\alpha 2)}}=(0.7,0.5,0.6599), F_{\mathrm{ts}_{(\beta 1)}}=(0.8,0.3,0.77)$, and $F_{\mathrm{ts}_{(\beta 2)}}=$ $(0.8,0.3,0.7699) \in \operatorname{SFS}(U)$ (from Example 1). Then, by Definition 13, we have

$$
\begin{aligned}
& \widehat{S} c\left(F_{\mathrm{ts}_{(\alpha 1)}}\right)=0.055504, \quad \widehat{S} c\left(F_{\mathrm{ts}_{(\alpha 2)}}\right)=0.055634, \\
& \widehat{S} c\left(F_{\mathrm{ts}_{(\beta 1)}}\right)=0.055467, \quad \widehat{S} c\left(F_{\mathrm{ts}_{(\beta 2)}}\right)=0.055644 \text {. }
\end{aligned}
$$

Since $\widehat{S} c\left(F_{\mathrm{ts}_{(\alpha 1)}}\right)<\widehat{S} c\left(F_{\mathrm{ts}_{(\beta 1)}}\right)$ and $\widehat{S}_{c}\left(F_{\mathrm{ts}_{(\alpha 2)}}\right)<\widehat{S}_{c}\left(F_{\left.\mathrm{ts}_{(\beta 2)}\right)}\right)$, $F_{\mathrm{ts}_{(\beta 1)}}>F_{\mathrm{ts}_{(\alpha 1)}}$ and $F_{\mathrm{ts}_{(\beta 2)}}>F_{\mathrm{ts}_{(\alpha 2)}}$.

Incorporating Examples 1 and 2, we can conclude that the new ranking framework is more reliable and less sensitive than in the previous process.

\section{Aggregation Operators Based on Yager's Norms}

This section presents some spherical fuzzy AOs using Yager OLs of SFNs. 


\subsection{Yager Weighted Averaging AOs}

Definition 15. Let $F_{g}=\left(\mu_{g}(v), \wp_{g}(v), \partial_{g}(v)\right) \in \operatorname{SFN}(U)$ $(g=1,2,3, \ldots, n)$. Then, Yager weighted averaging AOs for SFN $(U)$ are described as

$$
\operatorname{SFYWA}\left(F_{1}, F_{2}, \ldots, F_{n}\right)=\ell_{1} F_{1} \oplus \ell_{2} F_{2} \oplus \cdots \oplus \ell_{n} F_{n}=\sum_{g=1}^{n} \ell_{g} F_{g},
$$

where the weights $\left(\ell_{1}, \ell_{2}, \ldots, \ell_{g}\right)$ of $F_{g}$ have $\ell_{g} \geq 0$ and $\sum_{g=1}^{n} \ell_{g}=1$.

Theorem 4. Let $F_{g}=\left(\mu_{g}(v), \wp_{g}(v), \partial_{g}(v)\right) \in S F N(U)$ $(g=1,2,3, \ldots, n)$ and the weights $\left(\ell_{1}, \ell_{2}, \ldots, \ell_{g}\right)$ of $F_{g}$ have $\ell_{g} \geq 0$ and $\sum_{g=1}^{n} \ell_{g}=1$. The SFYWA AOs are a mapping $\mathscr{G}^{n} \longrightarrow \mathscr{G}$ such that

$$
\begin{aligned}
\operatorname{SFYWA}\left(F_{1}, F_{2}, \ldots, F_{n}\right) & =\sum_{g=1}^{n} \ell_{g} F_{g} \\
& =\left(\sqrt{\min \left(1,\left(\sum_{g=1}^{n} \ell_{g} \mu_{g}^{2 \delta}\right)^{1 / \delta}\right)}, \sqrt{1-\min \left(1,\left(\sum_{g=1}^{n} \ell_{g}\left(1-\wp_{g}^{2}\right)^{\delta}\right)^{1 / \delta}\right)},\right. \\
& \sqrt{1-\min \left(1,\left(\sum_{g=1}^{n} \ell_{g}\left(1-\partial_{g}^{2}\right)^{\delta}\right)^{1 / \delta}\right)} .
\end{aligned}
$$

Proof. We prove Theorem 4 by applying mathematical induction on $n$. For each $g, F_{g}=\left(\mu_{g}(v), \wp_{g}(v)\right.$, $\left.\partial_{g}(v)\right) \in$ SFN $(U)$, which implies that $\mu_{g}, \wp_{g}, \partial_{g} \in[0,1]$ and $\mu_{g}^{2}+\wp_{g}^{2}+\partial_{g}^{2} \leq 1$.

Step 1: for $n=2$, we get

$\operatorname{SFYWA}\left(F_{1}, F_{2}\right)=\ell_{1} F_{1} \oplus \ell_{2} F_{2}$

$$
\begin{aligned}
= & \left\{\sqrt{\min \left(1,\left(\ell_{1} \mu_{1}^{2 \delta}\right)^{1 / \delta}\right)}, \sqrt{1-\min \left(1,\left(\ell_{1}\left(1-\wp_{1}^{2}\right)^{\delta}\right)^{1 / \delta}\right)}, \sqrt{1-\min \left(1,\left(\ell_{1}\left(1-\partial_{1}^{2}\right)^{\delta}\right)^{1 / \delta}\right)}\right\} \\
& \oplus\left\{\sqrt{\min \left(1,\left(\ell_{2} \mu_{2}^{2 \delta}\right)^{1 / \delta}\right)}, \sqrt{1-\min \left(1,\left(\ell_{2}\left(1-\wp_{2}^{2}\right)^{\delta}\right)^{1 / \delta}\right)}, \sqrt{1-\min \left(1,\left(\ell_{2}\left(1-\partial_{2}^{2}\right)^{\delta}\right)^{1 / \delta}\right)}\right\} \\
= & \left(\sqrt{\min \left(1,\left(\sum_{g=1}^{2} \ell_{g} \mu_{g}^{2 \delta}\right)^{1 / \delta}\right)}, \sqrt{1-\min \left(1,\left(\sum_{g=1}^{2} \ell_{g}\left(1-\wp_{g}^{2}\right)^{\delta}\right)^{1 / \delta}\right)},\right. \\
& \left.\sqrt{1-\min \left(1,\left(\sum_{g=1}^{2} \ell_{g}\left(1-\partial_{g}^{2}\right)^{\delta}\right)^{1 / \delta}\right)}\right)
\end{aligned}
$$


Step 2: suppose that equation (22) holds for $n=\kappa$; we have

$$
\begin{aligned}
\operatorname{SFYWA}\left(F_{1}, F_{2}, \ldots, F_{\kappa}\right)= & \left(\sqrt{\min \left(1,\left(\sum_{g=1}^{\kappa} \ell_{g} \mu_{g}^{2 \delta}\right)^{1 / \delta}\right)}, \sqrt{1-\min \left(1,\left(\sum_{g=1}^{\kappa} \ell_{g}\left(1-\wp_{g}^{2}\right)^{\delta}\right)^{1 / \delta}\right)},\right. \\
& \sqrt{1-\min \left(1,\left(\sum_{g=1}^{\kappa} \ell_{g}\left(1-\partial_{g}^{2}\right)^{\delta}\right)^{1 / \delta}\right)} .
\end{aligned}
$$

Step 3: now, we have to prove that equation (22) holds

for $n=\kappa+1$.

$$
\begin{aligned}
\operatorname{SFYWA}\left(F_{1}, F_{2}, \ldots F_{\kappa+1}\right)= & \sum_{g=1}^{\kappa} \ell_{g} F_{g} \oplus \ell_{\kappa+1} F_{\kappa+1} \\
& =\left(\sqrt{\min \left(1,\left(\sum_{g=1}^{\kappa} \ell_{g} \mu_{g}^{2 \delta}\right)^{1 / \delta}\right)}, \sqrt{1-\min \left(1,\left(\sum_{g=1}^{\kappa} \ell_{g}\left(1-\wp_{g}^{2}\right)^{\delta}\right)^{1 / \delta}\right)},\right. \\
& \left.\sqrt{\left.1-\min \left(1,\left(\sum_{g=1}^{\kappa} \ell_{g}\left(1-\partial_{g}^{2}\right)^{\delta}\right)^{1 / \delta}\right)\right)}, \sqrt{1-\min \left(1,\left(\ell_{\kappa+1}\left(1-\wp_{\kappa+1}^{2}\right)^{\delta}\right)^{1 / \delta}\right)}, \sqrt{1-\min \left(1,\left(\ell_{\kappa+1}\left(1-\partial_{\kappa+1}^{2}\right)^{\delta}\right)^{1 / \delta}\right)}\right) \\
& \left(\sqrt{\min \left(1,\left(\ell_{\kappa+1} \mu_{\kappa+1}^{2 \delta}\right)^{1 / \delta}\right)}, \sqrt{\left.1,\left(\sum_{g=1}^{\kappa+1} \ell_{g} \mu_{g}^{2 \delta}\right)^{1 / \delta}\right), \sqrt{1-\min \left(1,\left(\sum_{g=1}^{\kappa+1} \ell_{g}\left(1-\wp_{g}^{2}\right)^{\delta}\right)^{1 / \delta}\right), \sqrt{1-\min \left(1,\left(\sum_{g=1}^{\kappa+1} \ell_{g}\left(1-\partial_{g}^{2}\right)^{\delta}\right)^{1 / \delta}\right)}}} .\right.
\end{aligned}
$$

That is, when $n=z+1$, equation (22) also holds.

Hence, equation (22) holds for any $n$. The proof is completed.

Next, we give some properties of the proposed SFYWA aggregation operator.
Theorem 5. Let $F_{g}=\left(\mu_{g}(v), \wp_{g}(v), \partial_{g}(v)\right) \in S F N(U)$ $(g=1,2,3, \ldots, n)$ such that $F_{g}=F$. Then,

$$
\operatorname{SFYWA}\left(F_{1}, F_{2}, \ldots, F_{n}\right)=F \text {. }
$$

Proof. Since $F_{g}=F(g=1,2,3, \ldots, n)$, by Theorem 4 , we get

$$
\begin{aligned}
\operatorname{SFYWA}\left(F_{1}, F_{2}, \ldots, F_{n}\right) & =\left(\sqrt{\min \left(1,\left(\sum_{g=1}^{n} \ell_{g} \mu_{g}^{2 \delta}\right)^{1 / \delta}\right)}, \sqrt{1-\min \left(1,\left(\sum_{g=1}^{n} \ell_{g}\left(1-\wp_{g}^{2}\right)^{\delta}\right)^{1 / \delta}\right)}, \sqrt{1-\min \left(1,\left(\sum_{g=1}^{n} \ell_{g}\left(1-\partial_{g}^{2}\right)^{\delta}\right)^{1 / \delta}\right)}\right. \\
& \left.=\left(\sqrt{\min \left(1,\left(\sum_{g=1}^{n} \ell_{g} \mu^{2 \delta}\right)^{1 / \delta}\right)}, \sqrt{1-\min \left(1,\left(\sum_{g=1}^{n} \ell_{g}\left(1-\wp^{2}\right)^{\delta}\right)^{1 / \delta}\right.}\right) \sqrt{1-\min \left(1,\left(\sum_{g=1}^{n} \ell_{g}\left(1-\partial^{2}\right)^{\delta}\right)^{1 / \delta}\right)}\right) \\
& =\left(\sqrt{\min \left(1,\left(\mu^{2 \delta}\right)^{1 / \delta}\right)}, \sqrt{1-\min \left(1,\left(\left(1-\wp^{2}\right)^{\delta}\right)^{1 / \delta}\right)}, \sqrt{1-\min \left(1,\left(\left(1-\partial^{2}\right)^{\delta}\right)^{1 / \delta}\right)}\right) \\
& =(\mu(v), \wp(v), \partial(v)) \\
& =F .
\end{aligned}
$$

The proof is completed. 
Theorem 6. Let $F_{g}=\left(\mu_{g}(v), \wp_{g}(v), \partial_{g}(v)\right), F_{g}^{-}=\{\min$ $\left.\left(\mu_{g}(v)\right), \min \left(\wp_{g}(v)\right), \max \left(\partial_{g}(v)\right)\right\}$, and $F_{g}^{+}=\left\{\max \left(\mu_{g}(v)\right)\right.$, $\left.\min \left(\wp_{g}(v)\right), \min \left(\partial_{g}(v)\right)\right\} \in S F N(U)(g=1,2,3, \ldots, n)$. Then,

$$
F_{g}^{-} \leq \operatorname{SFYWA}\left(F_{1}, F_{2}, \ldots, F_{n}\right) \leq F_{g}^{+} \text {. }
$$

Proof. The procedure is similar to the above theorem, so it is eliminated here.

Theorem 7. Let $F_{g}=\left(\mu_{g}(v), \wp_{g}(v), \partial_{g}(v)\right), F_{g}^{*}=\left(\mu_{g}^{*}(v)\right.$, $\left.\wp_{g}^{*}(v), \partial_{g}^{*}(v)\right) \in S F N(U)(g=1,2,3, \ldots, n)$. If $\mu_{g} \leq \mu_{g}^{*}, \wp_{g} \leq$ $\wp_{g}^{*}$ and $\partial_{g} \leq \partial_{g}^{*}$, then

$$
\operatorname{SFYWA}\left(F_{1}, F_{2}, \ldots, F_{n}\right) \leq \operatorname{SFYWA}\left(F_{1}^{*}, F_{2}^{*}, \ldots, F_{n}^{*}\right) \text {. }
$$

Proof. The procedure is similar to the above theorem, so it is eliminated here.
Definition 16. Let $F_{g}=\left(\mu_{g}(v), \wp_{g}(v), \partial_{g}(v)\right) \in \operatorname{SFN}(U)$ $(g=1,2,3, \ldots, n)$. Then, Yager ordered weighted averaging AOs for SFN $(U)$ are described as

$$
\begin{aligned}
\operatorname{SFYOWA}\left(F_{1}, F_{2}, \ldots, F_{n}\right) & =\ell_{1} F_{v(1)} \oplus \ell_{2} F_{v(2)} \oplus \cdots \oplus \ell_{n} F_{v(n)} \\
& =\sum_{g=1}^{n} \ell_{g} F_{v(g)},
\end{aligned}
$$

where $v(g)$ represented the ordered and $(v(1), v(2)$, $v(3), \ldots, v(n))$ is a permutation of $(1,2,3, \ldots, n)$, subject to $\varepsilon_{v(g-1)} \geq \varepsilon_{v(g)}$ for all $g$. Also the weights $\left(\ell_{1}, \ell_{2}, \ldots, \ell_{g}\right)$ of $F_{g}$ have $\ell_{g} \geq 0$ and $\sum_{g=1}^{n} \ell_{g}=1$.

Theorem 8. Let $F_{g}=\left(\mu_{g}(v), \wp_{g}(v), \partial_{g}(v)\right) \in S F N(U)$ $(g=1,2,3, \ldots, n)$ and the weights $\left(\ell_{1}, \ell_{2}, \ldots, \ell_{g}\right)$ of $F_{g}$ have $\ell_{g} \geq 0$ and $\sum_{g=1}^{n} \ell_{g}=1$. The SFYOWA AOs are a mapping $\mathscr{G}^{n} \longrightarrow \mathscr{G}$ such that

$$
\begin{aligned}
\operatorname{SFYOWA}\left(F_{1}, F_{2}, \ldots, F_{n}\right) & =\sum_{g=1}^{n} \ell_{g} F_{v(g)} \\
& =\left(\sqrt{\min \left(1,\left(\sum_{g=1}^{n} \ell_{g} \mu_{v(g)}^{2 \delta}\right)^{1 / \delta}\right)}, \sqrt{1-\min \left(1,\left(\sum_{g=1}^{n} \ell_{g}\left(1-\wp_{v(g)}^{2}\right)^{\delta}\right)\right.},\right. \\
& \left.\sqrt{1-\min \left(1,\left(\sum_{g=1}^{n} \ell_{g}\left(1-\partial_{v(g)}^{2}\right)^{\delta}\right)^{1 / \delta}\right)}\right)
\end{aligned}
$$

Proof. It follows from Theorem 4 similarly.

Theorem 9. Let $F_{g}=\left(\mu_{g}(v), \wp_{g}(v), \partial_{g}(v)\right) \in S F N(U)$ $(g=1,2,3, \ldots, n)$ such that $F_{g}=F$. Then,

$$
\operatorname{SFYOWA}\left(F_{1}, F_{2}, \ldots, F_{n}\right)=F \text {. }
$$

Theorem 10. Let $F_{g}=\left(\mu_{g}(v), \wp_{g}(v), \partial_{g}(v)\right), F_{g}^{-}=\left\{\min \left(\mu_{g}\right.\right.$ $\left.(v)), \min \left(\wp_{g}(v)\right), \max \left(\partial_{g}(v)\right)\right\}$, and $F_{g}^{+}=\left\{\max \left(\mu_{g}(v)\right)\right.$, $\left.\min \left(\wp_{g}(v)\right), \min \left(\partial_{g}(v)\right)\right\} \in S F N(U)(g=1,2,3, \ldots, n)$.

Then,

$$
F_{g}^{-} \leq \operatorname{SFYOWA}\left(F_{1}, F_{2}, \ldots, F_{n}\right) \leq F_{g}^{+}
$$

Theorem 11. Let $F_{g}=\left(\mu_{g}(v), \wp_{g}(v), \partial_{g}(v)\right), F_{g}^{*}=\left(\mu_{g}^{*}\right.$ $\left.(v), \wp_{g}^{*}(v), \partial_{g}^{*}(v)\right) \in S F N(U)(g=1,2,3, \ldots, n)$.

$\mu_{g} \leq \mu_{g}^{*}, \wp_{g} \leq \wp_{g}^{*}$ and $\partial_{g} \leq \partial_{g}^{*}$, then

$\operatorname{SFYOWA}\left(F_{1}, F_{2}, \ldots, F_{n}\right) \leq \operatorname{SFYOWA}\left(F_{1}^{*}, F_{2}^{*}, \ldots, F_{n}^{*}\right)$.

The proof of these theorems is similarly followed by Theorems 5-7.
Definition 17. Let $F_{g}=\left(\mu_{g}(v), \wp_{g}(v), \partial_{g}(v)\right) \in \operatorname{SFN}(U)$ $(g=1,2,3, \ldots, n)$. Then, Yager hybrid weighted averaging AOs for SFN $(U)$ are described as

$$
\begin{aligned}
\operatorname{SFYHWA}\left(F_{1}, F_{2}, \ldots, F_{n}\right) & =\sigma_{g} F_{v(1)}^{\prime} \oplus \sigma_{g} F_{v(2)}^{\prime} \oplus \cdots \oplus \sigma_{g} F_{v(n)}^{\prime} \\
& =\sum_{g=1}^{n} \sigma_{g} F_{v(g)}^{\prime},
\end{aligned}
$$

where weights $\left(\ell_{1}, \ell_{2}, \ldots, \ell_{g}\right)$ of $F_{g}$ have $\ell_{g} \geq 0$ and $\sum_{g=1}^{n} \ell_{g}=1$ and $g$ th biggest weighted value is $F_{v(g)}^{\prime}\left(F_{v(g)}^{\prime}=\right.$ $\left.n \ell_{g} F_{v(g)} \mid g=1,2, \ldots, n\right)$ consequently by total order $(v(1), v(2), v(3), \ldots, v(n))$. Also, associated weights $\left(\sigma_{1}, \sigma_{2}, \ldots, \sigma_{g}\right)$ of $F_{g}$ have $\sigma_{g} \geq 0$ and $\sum_{g=1}^{n} \sigma_{g}=1$.

Theorem 12. Let $F_{g}=\left(\mu_{g}(v), \wp_{g}(v), \partial_{g}(v)\right) \in S F N(U)$ $(g=1,2,3, \ldots, n)$ and the weights $\left(\ell_{1}, \ell_{2}, \ldots, \ell_{g}\right)$ of $F_{g}$ have $\ell_{g} \geq 0$ and $\sum_{g=1}^{n} \ell_{g}=1$. The SFYHWA AOs are a mapping $\mathscr{G}^{n} \longrightarrow \mathscr{G}$ with associated weights $\left(\sigma_{1}, \sigma_{2}, \ldots, \sigma_{g}\right)$ of $F_{g}$ having $\sigma_{g} \geq 0$ and $\sum_{g=1}^{n} \sigma_{g}=1$; we have 


$$
\begin{aligned}
\operatorname{SFYHWA}\left(F_{1}, F_{2}, \ldots, F_{n}\right) & =\sum_{g=1}^{n} \sigma_{g} F_{v(g)}^{\prime} \\
& =\left(\sqrt{\min \left(1,\left(\sum_{g=1}^{n} \ell_{g} \mu_{v(g)}^{\prime 2 \delta}\right)^{1 / \delta}\right)}, \sqrt{1-\min \left(1,\left(\sum_{g=1}^{n} \ell_{g}\left(1-\wp_{v(g)}^{\prime 2}\right)^{\delta}\right)^{1 / \delta}\right)}\right. \\
& \left.\sqrt{1-\min \left(1,\left(\sum_{g=1}^{n} \ell_{g}\left(1-\partial_{v(g)}^{\prime 2}\right)^{\delta}\right)^{1 / \delta}\right)}\right)
\end{aligned}
$$

Proof. It follows from Theorem 4 similarly.

Theorem 13. Let $F_{g}=\left(\mu_{g}(v), \wp_{g}(v), \partial_{g}(v)\right) \in S F N(U)$ $(g=1,2,3, \ldots, n)$ such that $F_{g}=F$. Then,

$$
\operatorname{SFYHWA}\left(F_{1}, F_{2}, \ldots, F_{n}\right)=F \text {. }
$$

\section{Theorem 14. Let $F_{g}=\left(\mu_{g}(v), \wp_{g}(v), \partial_{g}(v)\right)$,} $F_{g}^{-}=\left\{\min \left(\mu_{g}(v)\right), \min \left(\wp_{g}(v)\right), \max \left(\partial_{g}(v)\right)\right\}$, and $F_{g}^{+}=$ $\left\{\max \left(\mu_{g}(v)\right), \min \left(\wp_{g}(v)\right), \min \left(\partial_{g}(v)\right)\right\} \in S F N(U)(g=$ $1,2,3, \ldots, n)$. Then,

$$
F_{g}^{-} \leq \operatorname{SFYHWA}\left(F_{1}, F_{2}, \ldots, F_{n}\right) \leq F_{g}^{+} \text {. }
$$

Theorem 15. Let $F_{g}=\left(\mu_{g}(v), \wp_{g}(v), \partial_{g}(v)\right), F_{g}^{*}=$ $\left(\mu_{g}^{*}(v), \wp_{g}^{*}(v), \partial_{g}^{*}(v)\right) \in S F N(U)(g=1,2,3, \ldots, n)$. $\mu_{g} \leq \mu_{g}^{*}, \wp_{g} \leq \wp_{g}^{*}$ and $\partial_{g} \leq \partial_{g}^{*}$, then

$\operatorname{SFYHWA}\left(F_{1}, F_{2}, \ldots, F_{n}\right) \leq \operatorname{SFYHWA}\left(F_{1}^{*}, F_{2}^{*}, \ldots, F_{n}^{*}\right)$.
The proof of these theorems is similarly followed by Theorems 5-7.

\subsection{Yager Weighted Geometric AOs}

Definition 18. Let $F_{g}=\left(\mu_{g}(v), \wp_{g}(v), \partial_{g}(v)\right) \in \operatorname{SFN}(U)$ $(g=1,2,3, \ldots, n)$. Then, Yager weighted geometric AOs for SFN $(U)$ are described as

$$
\begin{aligned}
\operatorname{SFYWG}\left(F_{1}, F_{2}, \ldots, F_{n}\right) & =F_{1}^{\ell_{1}} \otimes F_{2}^{\ell_{2}} \otimes \cdots \otimes F_{n}^{\ell_{n}} \\
& =\prod_{g=1}^{n}\left(F_{g}\right)^{\ell_{g}},
\end{aligned}
$$

where the weights $\left(\ell_{1}, \ell_{2}, \ldots, \ell_{g}\right)$ of $F_{g}$ have $\ell_{g} \geq 0$ and $\sum_{g=1}^{n} \ell_{g}=1$.

Theorem 16. Let $F_{g}=\left(\mu_{g}(v), \wp_{g}(v), \partial_{g}(v)\right) \in S F N(U)$ $(g=1,2,3, \ldots, n)$ and the weights $\left(\ell_{1}, \ell_{2}, \ldots, \ell_{g}\right)$ of $F_{g}$ have $\ell_{g} \geq 0$ and $\sum_{g=1}^{n} \ell_{g}=1$. The SFYWG AOs are a mapping $\mathscr{G}^{n} \longrightarrow \mathscr{G}$ such that

$$
\begin{aligned}
\operatorname{SFYWG}\left(F_{1}, F_{2}, \ldots, F_{n}\right)= & \prod_{g=1}^{n}\left(F_{g}\right)^{\ell_{g}} \\
& =\left(\sqrt{1-\min \left(1,\left(\sum_{g=1}^{n} \ell_{g}\left(1-\mu_{g}^{2}\right)^{\delta}\right)^{1 / \delta}\right.}, \sqrt{1-\min \left(1,\left(\sum_{g=1}^{n} \ell_{g}\left(1-\wp_{g}^{2}\right)^{\delta}\right)^{1 / \delta}\right)}\right. \\
& \left.\sqrt{\min \left(1,\left(\sum_{g=1}^{n} \ell_{g} \partial_{g}^{2 \delta}\right)^{1 / \delta}\right)}\right)
\end{aligned}
$$

Proof. We prove Theorem 16 by applying mathematical induction on $n$. For each $g, F_{g}=\left(\mu_{g}(v), \wp_{g}(v)\right.$, $\left.\partial_{g}(v)\right) \in \operatorname{SFN}(U)$, which implies that $\mu_{g}, \wp_{g}, \partial_{g} \in[0,1]$ and $\mu_{g}^{2}+\wp_{g}^{2}+\partial_{g}^{2} \leq 1$.
Step 1: for $n=2$, we get

$$
\operatorname{SFYWG}\left(F_{1}, F_{2}\right)=F_{1}^{\ell_{1}} \otimes F_{2}^{\ell_{2}} \text {. }
$$


By Definition 12, we have

$\operatorname{SFYWG}\left(F_{1}, F_{2}\right)=F_{1}^{\ell_{1}} \otimes F_{2}^{\ell_{2}}$

$$
\begin{aligned}
& =\left\{\sqrt{1-\min \left(1,\left(\ell_{1}\left(1-\mu_{1}^{2}\right)^{\delta}\right)^{1 / \delta}\right)}, \sqrt{1-\min \left(1,\left(\ell_{1}\left(1-\wp_{1}^{2}\right)^{\delta}\right)^{1 / \delta}\right)}, \sqrt{\min \left(1,\left(\ell_{1} \partial_{1}^{2 \delta}\right)^{1 / \delta}\right)}\right\} \\
& \oplus\left\{\sqrt{1-\min \left(1,\left(\ell_{2}\left(1-\mu_{2}^{2}\right)^{\delta}\right)^{1 / \delta}\right)}, \sqrt{1-\min \left(1,\left(\ell_{2}\left(1-\wp_{2}^{2}\right)^{\delta}\right)^{1 / \delta}\right)}, \sqrt{\min \left(1,\left(\ell_{2} \partial_{2}^{2 \delta}\right)^{1 / \delta}\right)}\right\} \\
& =\left(\sqrt{1-\min \left(1,\left(\sum_{g=1}^{2} \ell_{g}\left(1-\mu_{g}^{2}\right)^{\delta}\right)^{1 / \delta}\right.}\right), \sqrt{1-\min \left(1,\left(\sum_{g=1}^{2} \ell_{g}\left(1-\wp_{g}^{2}\right)^{\delta}\right)^{1 / \delta}\right.}, \sqrt{\min \left(1,\left(\sum_{g=1}^{2} \ell_{g} \partial_{g}^{2 \delta}\right)^{1 / \delta}\right)} .
\end{aligned}
$$

Step 2: suppose that equation (42) holds for $n=\kappa$; we have

$$
\begin{aligned}
\operatorname{SFYWG}\left(F_{1}, F_{2}, \ldots, F_{\kappa}\right)= & \left(\sqrt{1-\min \left(1,\left(\sum_{g=1}^{\kappa} \ell_{g}\left(1-\mu_{g}^{2}\right)^{\delta}\right)^{1 / \delta}\right.}, \sqrt{1-\min \left(1,\left(\sum_{g=1}^{\kappa} \ell_{g}\left(1-\wp_{g}^{2}\right)^{\delta}\right)^{1 / \delta}\right)},\right. \\
& \sqrt{\min \left(1,\left(\sum_{g=1}^{\kappa} \ell_{g} \partial_{g}^{2 \delta}\right)^{1 / \delta}\right)} .
\end{aligned}
$$

Step 3: now, we have to prove that equation (42) holds for $n=\kappa+1$.

$$
\begin{aligned}
\operatorname{SFYWG}\left(F_{1}, F_{2}, \ldots F_{\kappa+1}\right)= & \prod_{g=1}^{\kappa}\left(F_{g}\right)^{\ell_{g}} \otimes\left(F_{\kappa+1}\right)^{\ell_{\kappa+1}} \\
= & \left(\sqrt{1-\min \left(1,\left(\sum_{g=1}^{\kappa} \ell_{g}\left(1-\mu_{g}^{2}\right)^{\delta}\right)^{1 / \delta}\right)}, \sqrt{1-\min \left(1,\left(\sum_{g=1}^{\kappa} \ell_{g}\left(1-\wp_{g}^{2}\right)^{\delta}\right)^{1 / \delta}\right)}, \sqrt{\min \left(1,\left(\sum_{g=1}^{\kappa} \ell_{g} \partial_{g}^{2 \delta}\right)\right.}\right) \\
& \left.\oplus \sqrt{1-\min \left(1,\left(\ell_{\kappa+1}\left(1-\mu_{\kappa+1}^{2}\right)^{\delta}\right)^{1 / \delta}\right)}, \sqrt{1-\min \left(1,\left(\ell_{\kappa+1}\left(1-\wp_{\kappa+1}^{2}\right)^{\delta}\right)^{1 / \delta}\right)}, \sqrt{\min \left(1,\left(\ell_{\kappa+1} \partial_{\kappa+1}^{2 \delta}\right)^{1 / \delta}\right)}\right\} \\
= & \left(\sqrt{1-\min \left(1,\left(\sum_{g=1}^{\kappa+1} \ell_{g}\left(1-\mu_{g}^{2}\right)^{\delta}\right)^{1 / \delta}\right)}, \sqrt{1-\min \left(1,\left(\sum_{g=1}^{\kappa+1} \ell_{g}\left(1-\wp_{g}^{2}\right)^{\delta}\right)^{1 / \delta}\right.}\right), \\
& \sqrt{\min \left(1,\left(\sum_{g=1}^{\kappa+1} \ell_{g} \partial_{g}^{2 \delta}\right)^{1 / \delta}\right)} .
\end{aligned}
$$

That is, when $n=z+1$, equation (42) also holds.

Hence, equation (42) holds for any $n$. The proof is completed.
Theorem 17. Let $F_{g}=\left(\mu_{g}(v), \wp_{g}(v), \partial_{g}(v)\right) \in S F N(U)$ $(g=1,2,3, \ldots, n)$ such that $F_{g}=F$. Then, 


$$
\operatorname{SFYWG}\left(F_{1}, F_{2}, \ldots, F_{n}\right)=F \text {. }
$$

Proof. Since $F_{g}=F(g=1,2,3, \ldots, n)$, by Theorem 16, we get

$$
\begin{aligned}
\operatorname{SFYWG}\left(F_{1}, F_{2}, \ldots, F_{n}\right) & =\left(\sqrt{1-\min \left(1,\left(\sum_{g=1}^{n} \ell_{g}\left(1-\mu^{2}\right)^{\delta}\right)^{1 / \delta}\right)}, \sqrt{1-\min \left(1,\left(\sum_{g=1}^{n} \ell_{g}\left(1-\wp^{2}\right)^{\delta}\right)^{1 / \delta}\right)},\right. \\
& \left.\sqrt{\min \left(1,\left(\sum_{g=1}^{n} \ell_{g} \partial^{2 \delta}\right)^{1 / \delta}\right)}\right) \\
& =\left(\sqrt{1-\min \left(1,\left(1-\mu^{2}\right)\right)}, \sqrt{1-\min \left(1,\left(1-\wp^{2}\right)\right)}, \sqrt{\min \left(1,\left(\partial^{2}\right)\right)}\right) \\
& =(\mu(v), \wp(v), \partial(v)) \\
& =F .
\end{aligned}
$$

The proof is completed.

Theorem 18. Let $F_{g}=\left(\mu_{g}(v), \wp_{g}(v), \partial_{g}(v)\right), F_{g}^{-}=$ $\left\{\min \left(\mu_{g}(v)\right), \min \left(\wp_{g}(v)\right), \max \left(\partial_{g}(v)\right)\right\}$ and $F_{g}^{+}=$ $\left\{\max \left(\mu_{g}(v)\right), \min \left(\wp_{g}(v)\right), \min \left(\partial_{g}(v)\right)\right\} \in S F N(U) \quad(g=$ $1,2,3, \ldots, n)$. Then,

$$
F_{g}^{-} \leq \operatorname{SFYWG}\left(F_{1}, F_{2}, \ldots, F_{n}\right) \leq F_{g}^{+} .
$$

Theorem 19. Let $F_{g}=\left(\mu_{g}(v), \wp_{g}(v), \partial_{g}(v)\right), F_{g}^{*}=$

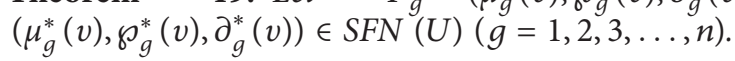

$\mu_{g} \leq \mu_{g}^{*}, \wp_{g} \leq \wp_{g}^{*}$ and $\partial_{g} \leq \partial_{g}^{*}$, then

$\operatorname{SFYWG}\left(F_{1}, F_{2}, \ldots, F_{n}\right) \leq \operatorname{SFYWG}\left(F_{1}^{*}, F_{2}^{*}, \ldots, F_{n}^{*}\right)$.

Definition 19. Let $F_{g}=\left(\mu_{g}(v), \wp_{g}(v), \partial_{g}(v)\right) \in \operatorname{SFN}(U)$ $(g=1,2,3, \ldots, n)$. Then, Yager ordered weighted geometric AOs for SFN $(U)$ are described as

$$
\operatorname{SFYOWG}\left(F_{1}, F_{2}, \ldots, F_{n}\right)=\left(F_{v(1)}\right)^{\ell_{1}} \otimes\left(F_{v(2)}\right)^{\ell_{2}} \otimes \cdots \otimes\left(F_{v(n)}\right)^{\ell_{n}}=\prod_{g=1}^{n}\left(F_{v(g)}\right)^{\ell_{g}},
$$

where $v(g)$ represented the ordered and $(v(1), v(2), v(3), \ldots, v(n))$ is a permutation of $(1,2,3, \ldots, n)$, subject to $\varepsilon_{v(g-1)} \geq \varepsilon_{v(g)}$ for all $g$. Also the weights $\left(\ell_{1}, \ell_{2}, \ldots, \ell_{g}\right)$ of $F_{g}$ have $\ell_{g} \geq 0$ and $\sum_{g=1}^{n} \ell_{g}=1$.
Theorem 20. Let $F_{g}=\left(\mu_{g}(v), \wp_{g}(v), \partial_{g}(v)\right) \in S F N(U)$ $(g=1,2,3, \ldots, n)$ and the weights $\left(\ell_{1}, \ell_{2}, \ldots, \ell_{g}\right)$ of $F_{g}$ have $\ell_{g} \geq 0$ and $\sum_{g=1}^{n} \ell_{g}=1$. The SFYOWG AOs are a mapping $\mathscr{G}^{n} \longrightarrow \mathscr{G}$ such that

$$
\begin{aligned}
\operatorname{SFYOWG}\left(F_{1}, F_{2}, \ldots, F_{n}\right)=\prod_{g=1}^{n}\left(F_{v(g)}\right)^{\ell_{g}} & =\left(\sqrt{1-\min \left(1,\left(\sum_{g=1}^{n} \ell_{g}\left(1-\mu_{v(g)}^{2}\right)^{\delta}\right)^{1 / \delta}\right)},\right. \\
& \sqrt{1-\min \left(1,\left(\sum_{g=1}^{n} \ell_{g}\left(1-\wp_{v(g)}^{2}\right)^{\delta}\right)^{1 / \delta}\right)}, \sqrt{\min \left(1,\left(\sum_{g=1}^{n} \ell_{g} \partial_{v(g)}^{2 \delta}\right)^{1 / \delta}\right)} .
\end{aligned}
$$

Proof. It follows from Theorem 16 similarly.

Theorem 21. Let $F_{g}=\left(\mu_{g}(v), \wp_{g}(v), \partial_{g}(v)\right) \in S F N(U)$ $(g=1,2,3, \ldots, n)$ such that $F_{g}=F$. Then,

$$
\operatorname{SFYOWG}\left(F_{1}, F_{2}, \ldots, F_{n}\right)=F \text {. }
$$

Theorem 22. Let $F_{g}=\left(\mu_{g}(v), \wp_{g}(v), \partial_{g}(v)\right), F_{g}^{-}=$ $\left\{\min \left(\mu_{g}(v)\right), \min \left(\wp_{g}(v)\right), \max \left(\partial_{g}(v)\right)\right\}$ and $F_{g}^{+}=$ $\left\{\max \left(\mu_{g}(v)\right), \min \left(\wp_{g}(v)\right), \min \left(\partial_{g}(v)\right)\right\} \in S F N(U) \quad(g=$ $1,2,3, \ldots, n)$. Then,

$$
F_{g}^{-} \leq \operatorname{SFYOWG}\left(F_{1}, F_{2}, \ldots, F_{n}\right) \leq F_{g}^{+} .
$$


Theorem 23. Let $F_{g}=\left(\mu_{g}(v), \wp_{g}(v), \partial_{g}(v)\right), F_{g}^{*}=\left(\mu_{g}^{*}(v)\right.$, $\left.\wp_{g}^{*}(v), \partial_{g}^{*}(v)\right) \in \operatorname{SFN}^{(U)}(g=1,2,3, \ldots, n)$. If $\mu_{g} \leq \mu_{g}^{*}, \wp_{g} \leq$ $\wp_{g}^{*}$ and $\partial_{g} \leq \partial_{g}^{*}$, then

$\operatorname{SFYOWG}\left(F_{1}, F_{2}, \ldots, F_{n}\right) \leq \operatorname{SFYOWG}\left(F_{1}^{*}, F_{2}^{*}, \ldots, F_{n}^{*}\right)$.
The proof of these theorems is similarly followed by Theorems 17-19.

Definition 20. Let $F_{g}=\left(\mu_{g}(v), \wp_{g}(v), \partial_{g}(v)\right) \in \operatorname{SFN}(U)$ $(g=1,2,3, \ldots, n)$. Then, Yager hybrid weighted geometric AOs for SFN $(U)$ are described as

$$
\operatorname{SFYHWG}\left(F_{1}, F_{2}, \ldots, F_{n}\right)=\left(F_{v(1)}^{\prime}\right)^{\sigma_{g}} \otimes\left(F_{v(2)}^{\prime}\right)^{\sigma_{g}} \otimes \cdots \otimes\left(F_{v(n)}^{\prime}\right)^{\sigma_{g}}=\prod_{g=1}^{n}\left(F_{v(g)}\right)^{\prime}
$$

where weights $\left(\ell_{1}, \ell_{2}, \ldots, \ell_{g}\right)$ of $F_{g}$ have $\ell_{g} \geq 0$ and $\sum_{g=1}^{n} \ell_{g}=1$ and $g$ th biggest weighted value is $F_{v(g)}\left(F_{v(g)}{ }^{\prime}=\right.$ $\left.n \ell_{g} F_{v(g)} \mid g=1,2, \ldots, n\right)$ consequently by total order $(v(1), v(2), v(3), \ldots, v(n))$. Also, associated weights $\left(\sigma_{1}, \sigma_{2}, \ldots, \sigma_{g}\right)$ of $F_{g}$ have $\sigma_{g} \geq 0$ and $\sum_{g=1}^{n} \sigma_{g}=1$.
Theorem 24. Let $F_{g}=\left(\mu_{g}(v), \wp_{g}(v), \partial_{g}(v)\right) \in \operatorname{SFN}(U)$ $(g=1,2,3, \ldots, n)$ and the weights $\left(\ell_{1}, \ell_{2}, \ldots, \ell_{g}\right)$ of $F_{g}$ have $\ell_{g} \geq 0$ and $\sum_{g=1}^{n} \ell_{g}=1$. The SFYHWG AOs are a mapping $\mathscr{G}^{n} \longrightarrow \mathscr{G}$ with associated weights $\left(\sigma_{1}, \sigma_{2}, \ldots, \sigma_{g}\right)$ of $F_{g}$ having $\sigma_{g} \geq 0$ and $\sum_{g=1}^{n} \sigma_{g}=1$; we have

$$
\begin{aligned}
\operatorname{SFYHWG}\left(F_{1}, F_{2}, \ldots, F_{n}\right) & =\prod_{g=1}^{n}\left(F_{v(g)}^{\prime}\right)^{\sigma_{g}}= \\
= & \sqrt{1-\min \left(1,\left(\sum_{g=1}^{n} \ell_{g}\left(1-\mu_{v(g)}^{\prime 2}\right)^{\delta}\right)^{1 / \delta}\right.},
\end{aligned}
$$

Proof. It follows from Theorem 16 similarly.

Theorem 25. Let $F_{g}=\left(\mu_{g}(v), \wp_{g}(v), \partial_{g}(v)\right) \in S F N(U)$ $(g=1,2,3, \ldots, n)$ such that $F_{g}=F$. Then,

$$
\operatorname{SFYHWG}\left(F_{1}, F_{2}, \ldots, F_{n}\right)=F \text {. }
$$

Theorem 26. Let $F_{g}=\left(\mu_{g}(v), \wp_{g}(v), \partial_{g}(v)\right), F_{g}^{-}=\{\min$ $\left.\left(\mu_{g}(v)\right), \min \left(\wp_{g}(v)\right), \max \left(\partial_{g}(v)\right)\right\}$, and $F_{g}^{+}=\left\{\max \left(\mu_{g}(v)\right)\right.$, $\left.\min \left(\wp_{g}(v)\right), \min \left(\partial_{g}(v)\right)\right\} \in S F N(U)(g=1,2,3, \ldots, n)$. Then,

$$
F_{g}^{-} \leq \operatorname{SFYHWG}\left(F_{1}, F_{2}, \ldots, F_{n}\right) \leq F_{g}^{+} .
$$

Theorem 27. Let $F_{g}=\left(\mu_{g}(v), \wp_{g}(v), \partial_{g}(v)\right), F_{g}^{*}=\left(\mu_{g}^{*}\right.$ $\left.(v), \wp_{g}^{*}(v), \partial_{g}^{*}(v)\right) \in S F N(U)(g=1,2,3, \ldots, n)$. If $\mu_{g} \leq \mu_{g}^{*}$, $\wp_{g} \leq \wp_{g}^{*}$ and $\partial_{g} \leq \partial_{g}^{*}$, then

$$
\operatorname{SFYHWG}\left(F_{1}, F_{2}, \ldots, F_{n}\right) \leq \operatorname{SFYHWG}\left(F_{1}^{*}, F_{2}^{*}, \ldots, F_{n}^{*}\right) \text {. }
$$

The proof of these theorems is similarly followed by Theorems 17-19.

\section{Algorithm for Decision-Making Problems (DMPs)}

In this section, we propose a framework for solving multiattribute DMPs under SF information. Consider a MAGDM with a set of $m$ alternatives $\left\{\beth_{1}, \beth_{2}, \ldots, \beth_{g}\right\}$ and let $\left.\left\{\neg_{1}, \neg_{2}, \ldots,\right\urcorner_{h}\right\}$ be a set of attributes with weight vector $\ell=\left(\ell_{1}, \ell_{2}, \ldots, \ell_{h}\right)$, where $\ell_{t} \in[0,1]$ and $\sum_{t=1}^{h} \ell_{t}=1$. To assess the performance of $k$ th alternative $\beth_{k}$ under the $t$-th

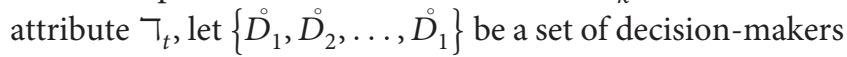
and let $\widehat{w}=\left(\widehat{w}_{1}, \widehat{w}_{2}, \ldots, \widehat{w}_{\hat{j}}\right)$ be the weighted vector of decision-makers with $\widehat{w}_{s} \in[0,1]$ and $\sum_{s=1}^{\hat{j}} \widehat{w}_{s}=1$. The SF decision matrix can be written as 


$$
\left[\begin{array}{cccc}
\left(\mu_{11}(v), \wp_{11}(v), \partial_{11}(v)\right) & \left(\mu_{12}(v), \wp_{12}(v), \partial_{12}(v)\right) & \cdots & \left(\mu_{1 h}(v), \wp_{1 h}(v), \partial_{1 h}(v)\right) \\
\left(\mu_{21}(v), \wp_{21}(v), \partial_{21}(v)\right) & \left(\mu_{22}(v), \wp_{22}(v), \partial_{22}(v)\right) & \cdots & \left(\mu_{2 h}(v), \wp_{2 h}(v), \partial_{2 h}(v)\right) \\
\left(\mu_{31}(v), \wp_{31}(v), \partial_{31}(v)\right) & \left(\mu_{32}(v), \wp_{32}(v), \partial_{32}(v)\right) & \cdots & \left(\mu_{3 h}(v), \wp_{3 h}(v), \partial_{3 h}(v)\right) \\
\vdots & \vdots & \ddots & \vdots \\
\left(\mu_{g 1}(v), \wp_{g 1}(v), \partial_{g 1}(v)\right) & \left(\mu_{g 2}(v), \wp_{g 2}(v), \partial_{g 2}(v)\right) & \cdots & \left(\mu_{g h}(v), \wp_{g h}(v), \partial_{g h}(v)\right)
\end{array}\right],
$$

where $\mu(v) \in[0,1], \wp(v) \in[0,1]$, and $\partial(v) \in[0,1]$ are positive, neutral, and negative membership grades, respectively. In addition, $0 \leq \mu^{2}(v)+\wp^{2}(v)+\partial^{2}(v) \leq 1, \forall v \in U$. Key steps of the developed multiattribute group decisionmaking (MAGDM) problem are described as follows:
Step 1: construct the SF decision matrix based on the experts evaluations.

$$
\left[\begin{array}{cccc}
\left(\mu_{11}^{\widehat{j}}(v), \wp_{11}^{\widehat{j}}(v), \partial_{11}^{\widehat{j}}(v)\right) & \left(\mu_{12}^{\widehat{j}}(v), \wp_{12}^{\widehat{j}}(v), \partial_{12}^{\widehat{j}}(v)\right) & \cdots & \left(\mu_{1 h}^{\widehat{j}}(v), \wp_{1 h}^{\widehat{j}}(v), \partial_{1 h}^{\widehat{j}}(v)\right) \\
\left(\mu_{21}^{\widehat{j}}(v), \wp_{21}^{\widehat{j}}(v), \partial_{21}^{\widehat{j}}(v)\right) & \left(\mu_{22}^{\widehat{j}}(v), \wp_{22}^{\widehat{j}}(v), \partial_{22}^{\widehat{j}}(v)\right) & \cdots & \left(\mu_{2 h}^{\widehat{j}}(v), \wp_{2 h}^{\widehat{j}}(v), \partial_{2 h}^{\widehat{j}}(v)\right) \\
\left(\mu_{31}^{\widehat{j}}(v), \wp_{31}^{\widehat{j}}(v), \partial_{31}^{\widehat{j}}(v)\right) & \left(\mu_{32}^{\widehat{j}}(v), \wp_{32}^{\widehat{j}}(v), \partial_{32}^{\widehat{j}}(v)\right) & \cdots & \left(\mu_{3 h}^{\widehat{j}}(v), \wp_{3 h}^{\widehat{j}}(v), \partial_{3 h}^{\widehat{j}}(v)\right) \\
\vdots & \ddots & \vdots \\
\left(\mu_{g 1}^{\widehat{j}}(v), \wp_{g 1}^{\widehat{j}}(v), \partial_{g 1}^{\hat{j}}(v)\right) & \left(\mu_{g 2}^{\widehat{j}}(v), \wp_{g 2}^{\widehat{j}}(v), \partial_{g 2}^{\widehat{j}}(v)\right) & \cdots & \left(\mu_{g h}^{\widehat{j}}(v), \wp_{g h}^{\widehat{j}}(v), \partial_{g h}^{\widehat{j}}(v)\right)
\end{array}\right],
$$

where $\hat{j}$ represents the number of experts.

Step 2: aggregate the individual decision matrices based on the aggregation operators to construct the aggregated matrix. Hence, the aggregated decision matrix is constructed as

$$
\left[\begin{array}{cccc}
\left(\mu_{11}(v), \wp_{11}(v), \partial_{11}(v)\right) & \left(\mu_{12}(v), \wp_{12}(v), \partial_{12}(v)\right) & \cdots & \left(\mu_{1 h}(v), \wp_{1 h}(v), \partial_{1 h}(v)\right) \\
\left(\mu_{21}(v), \wp_{21}(v), \partial_{21}(v)\right) & \left(\mu_{22}(v), \wp_{22}(v), \partial_{22}(v)\right) & \cdots & \left(\mu_{2 h}(v), \wp_{2 h}(v), \partial_{2 h}(v)\right) \\
\left(\mu_{31}(v), \wp_{31}(v), \partial_{31}(v)\right) & \left(\mu_{32}(v), \wp_{32}(v), \partial_{32}(v)\right) & \cdots & \left(\mu_{3 h}(v), \wp_{3 h}(v), \partial_{3 h}(v)\right) \\
\vdots & \vdots & \ddots & \vdots \\
\left(\mu_{g 1}(v), \wp_{g 1}(v), \partial_{g 1}(v)\right) & \left(\mu_{g 2}(v), \wp_{g 2}(v), \partial_{g 2}(v)\right) & \cdots & \left(\mu_{g h}(v), \wp_{g h}(v), \partial_{g h}(v)\right)
\end{array}\right]
$$

Step 3: if the weights of the attribute are known as a prior, then use them. Otherwise, we will calculate them using the concept of spherical entropy measure. Spherical entropy measure is as follows:

$$
\ell_{j}=\frac{1+(1 / h) \sum_{i=1}^{h}\left(\mu_{i j} \log \left(\mu_{i j}\right)+\wp_{i j} \log \left(\wp_{i j}\right)+\partial_{i j} \log \left(\partial_{i j}\right)\right)}{\sum_{j=1}^{g}\left(1+(1 / h) \sum_{i=1}^{h}\left(\mu_{i j} \log \left(\mu_{i j}\right)+\wp_{i j} \log \left(\wp_{i j}\right)+\partial_{i j} \log \left(\partial_{i j}\right)\right)\right)} .
$$

Step 3: exploit the established aggregation operators to achieve the SFN $F_{t}(t=1,2, \ldots, g)$ for the alternatives $I_{k}$, that is, the established operators to obtain the collective overall preference values of
$F_{t}(t=1,2, \ldots, g)$ for the alternatives $\beth_{k}$, where $\ell=$ $\left(\ell_{1}, \ell_{2}, \ldots, \ell_{h}\right)$ is the weight vector of the attributes.

Step 4: after that, we compute the scores of all the overall values $F_{t}(t=1,2, \ldots, g)$ for the alternatives $\beth_{k}$. 
Step 5: according to Definition 13, rank the alternatives $\beth_{k}(k=1,2, \ldots, g)$ and select the best one having the greater value.

\section{Application of Proposed Decision- Making Technique}

In this section, a numerical application about wind power plant location selection problem is firstly used to illustrate the designed MAGDM method. Then a comparison between the presented Yager aggregation operators and the existing aggregation operators of spherical fuzzy numbers is carried out to show the characteristics and advantage of the presented AOs.

7.1. Practical Case Study. In this segment, a case study is provided to illustrate the effectiveness and reliability of the established decision-making approach.

The case study area was Jhimpir, a village in Thatta district of Sindh province in Pakistan, 120 kilometres northeast of Karachi. Jhimpir's geographical coordinates are $25^{\circ} 1^{\prime} 0^{\prime \prime}$ North, $68^{\circ} 1^{\prime} 0^{\prime \prime}$ East. Location of Jhimpir is shown in Figure 1.

The digital elevation model of Jhimpir is given in Figure 2.The required data were collected from numerous resources including governmental agencies, open sources, and related literature such as National Authority for Remote Sensing \& Space Sciences, Pakistan Meteorological Authority, New and Renewable Energy Authority, Pakistan General Survey Authority, NASA's Prediction of Worldwide Energy Resources (POWER), United States Geological Survey, and Pakistan Environmental Affairs Agency.

Electricity plays an essential part in any nation's socioeconomic progress and social prosperity. Electricity energy should be regarded as the fundamental need for human development. In Pakistan, limited power generation is a major issue that directly restricts the country's growth. In a landmark achievement, the 50-megawatt Jhimpir wind power project has begun commercial operations as Pakistan gradually moves to ramp up renewable energy generation in line with the global trend and to bridge the domestic shortfall. The total cost of project is $\$ 136$ million. Completed in 2002, it has a total capacity of $50 \mathrm{MW}$. This wind corridor has a 50000-megawatt potential with average wind speeds over 7 meters per second. The government has announced upfront tariff and ROI of 17 percent, which is highest in the world. There are 14 projects in the pipeline, out of which 50 MW FFCEL project has achieved COD by mid-December 2012.

Pakistan's National Renewable Energy Laboratories (NREL) wind resource map has provided a major boost to the development of wind power in the wind corridor regions. These regions are the Karachi-Hyderabad region especially on hilltops, ridges in the northern Indus valley, wind corridor areas in western Pakistan, high mountainous regions, and hills and ridges in southwestern Pakistan. This potential area has now become the focal point of wind power 's near future development. The coastal belt of Pakistan has a wind corridor that is $60 \mathrm{~km}$ wide and $180 \mathrm{~km}$ long, as per the collected data. This corridor has an electricity generation potential of up to $50000 \mathrm{MW}$ of exploitable wind power. Here, we enlist the wind power energy projects and discuss their production in Table 1.

For our research, we used a dataset comprising topographic, geological, and climatic factors. Based on several literatures and case studies concerning wind farm site selection and local conditions, different criteria were reviewed by three experts and five locations $\left\{I_{1}, I_{2}, I_{3}, I_{4}, I_{5}\right\}$ under four criteria were selected to evaluate the suitable sites for wind farms. The detailed description of the criteria is as follows:

(1) Natural Factors $\left(\neg_{1}\right)$ : in order to be effective, the position of the wind turbines is measured by the prevailing wind direction. In generating electricity from wind turbines, wind speed is a vital factor. Wind speed above certain rates is essential for producing wind energy $[50,51]$.

(2) Political Aspect $\left(\neg_{2}\right)$ : select the location that offers maximum output, minimizing project costs, giving the political point score to the government for installations of wind energy projects.

(3) Socioeconomic Factors $\left(T_{3}\right)$ : in order to minimize the cost of building wind farms and to reduce the cost of transporting electricity, wind farms should be located close to the existing transmission grids [52].

(4) Environmental Factors $\left(\neg_{4}\right)$ : wind farms in areas where they negligibly interfere with existing land use outside protected areas, artificial surfaces, wetlands, aquatic areas, and forestry areas should be installed [52]. It is necessary to keep all the mechanical parts of wind turbines away from the water. Wind turbine fins are lowered and disconnected to prevent harm to the components of the turbine.

The three experts were asked in this assessment to use spherical fuzzy information and their weights are $(0.314,0.355,0.331)^{T}$.

Step 1: three experts listed their evaluation information using the spherical fuzzy numbers in Tables 2-4

Step 2: aggregated SF information is evaluated using spherical fuzzy weighted averaging operators. The results are shown in Table 5.

Step 4: now, we find out the attribute weight vector using spherical fuzzy entropy measure as follows.

$$
\ell=\left\{\ell_{1}=0.256, \ell_{2}=0.248, \ell_{3}=0.245, \ell_{4}=0.251\right\} \text {. }
$$

Step 5: evaluate the overall perfumes of the alternatives; we utilized the proposed Yager aggregation operators as follows in Tables 6 and 7 .

Step 6: compute the score value of each collective SF information of each alternative as follows in Table 8 . 


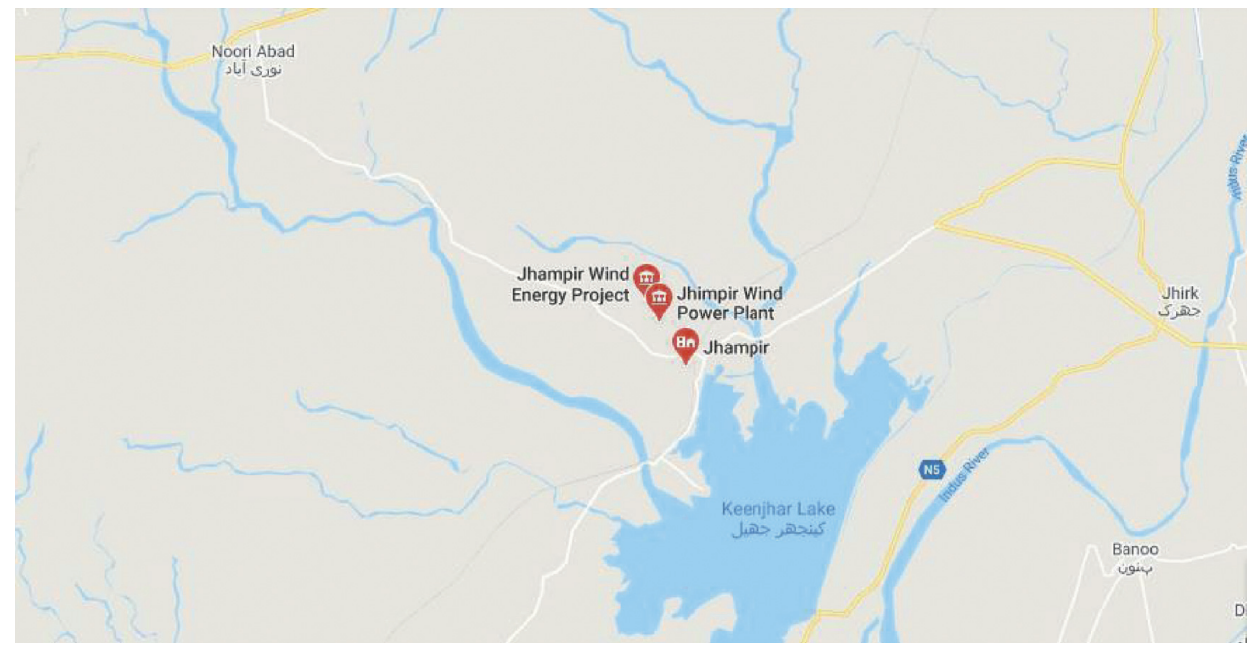

FIgURE 1: Location of Jhimpir.

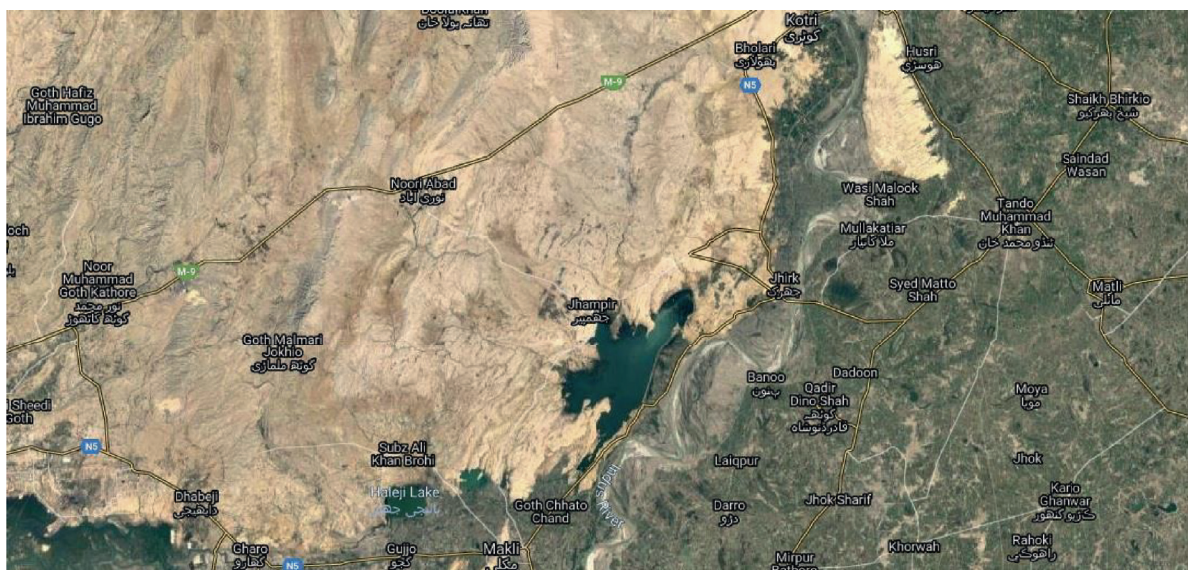

Figure 2: Digital elevation model.

TABLE 1: Wind power projects and their production.

\begin{tabular}{|c|c|c|c|}
\hline Station & Location & Capacity (MW) & In service date \\
\hline FFC Energy Wind Project & Jhimpir, Sindh & 49.5 & 2013 \\
\hline Zorlu Enerji Pakistan & Jhimpir, Sindh & 56.4 & 2013 \\
\hline Three Gorges Pvt. Ltd. & Jhimpir, Sindh & 150 & 2014 \\
\hline Sapphire Wind Power Pvt. Ltd. & Jhimpir, Sindh & 52.8 & 2015 \\
\hline Yunus Energy Ltd. & Jhimpir, Sindh & 50 & 2016 \\
\hline Metro Wind Power Co. Ltd. & Jhimpir, Sindh & 50 & 2016 \\
\hline Gul Ahmed Wind Power Ltd. & Jhimpir, Sindh & 50 & 2016 \\
\hline Master Wind Energy Ltd. & Jhimpir, Sindh & 52.8 & 2016 \\
\hline ACT Wind Pvt. Ltd. & Jhimpir, Sindh & 30 & 2016 \\
\hline Sachal Energy Wind Farm & Jhimpir, Sindh & 50 & 2017 \\
\hline United Energy Pakistan Wind Ltd. & Jhimpir, Sindh & 100 & 2017 \\
\hline Hawa Energy Ltd. & Jhimpir, Sindh & 50 & 2018 \\
\hline Burj Capital Jhimpir Wind Power Limited & Jhimpir, Sindh & 50 & 2018 \\
\hline Artistic Energy Pvt. Ltd. & Jhimpir, Sindh & 49.3 & 2018 \\
\hline Tricon Boston Corporation & Jhimpir, Sindh & 150 & 2018 \\
\hline
\end{tabular}

TABle 2: $D_{1}$.

\begin{tabular}{lcccr}
\hline & $\neg_{1}$ & $\neg_{2}$ & \urcorner$_{3}$ & $\neg_{4}$ \\
\hline$\lrcorner_{1}$ & $(0.84,0.34,0.40)$ & $(0.78,0.39,0.43)$ & $(0.67,0.50,0.30)$ & $(0.71,0.21,0.31)$ \\
\lrcorner$_{2}$ & $(0.60,0.11,0.53)$ & $(0.59,0.35,0.23)$ & $(0.72,0.31,0.41)$ & $(0.82,0.25,0.11)$ \\
\lrcorner$_{3}$ & $(0.79,0.19,0.39)$ & $(0.91,0.21,0.11)$ & $(0.71,0.41,0.13)$ & $(0.51,0.25,0.34)$ \\
\lrcorner$_{4}$ & $(0.63,0.51,0.13)$ & $(0.42,0.33,0.49)$ & $(0.61,0.43,0.45)$ & $(0.59,0.37,0.49)$ \\
$I_{5}$ & $(0.57,0.36,0.29)$ & $(0.60,0.15,0.50)$ & $(0.70,0.32,0.40)$ & $(0.65,0.44,0.33)$ \\
\hline
\end{tabular}


TABle 3: $D_{2}$.

\begin{tabular}{|c|c|c|c|c|}
\hline & \urcorner$_{1}$ & $7_{2}$ & $7_{3}$ & $7_{4}$ \\
\hline$I_{1}$ & $(0.61,0.15,0.53)$ & $(0.62,0.35,0.16)$ & $(0.61,0.35,0.47)$ & $(0.74,0.17,0.55)$ \\
\hline$I_{2}$ & $(0.66,0.11,0.51)$ & $(0.77,0.23,0.43)$ & $(0.93,0.08,0.09)$ & $(0.99,0.06,0.02)$ \\
\hline$I_{3}$ & $(0.88,0.09,0.07)$ & $(0.89,0.06,0.05)$ & $(0.56,0.17,0.44)$ & $(0.61,0.13,0.43)$ \\
\hline$z_{4}$ & $(0.59,0.32,0.34)$ & $(0.51,0.48,0.24)$ & $(0.68,0.53,0.39)$ & $(0.61,0.21,0.34)$ \\
\hline$\beth_{5}$ & $(0.71,0.31,0.24)$ & $(0.69,0.41,0.35)$ & $(0.73,0.44,0.21)$ & $(0.74,0.49,0.22)$ \\
\hline
\end{tabular}

TABle 4: $D_{3}$.

\begin{tabular}{lcccr}
\hline & $\neg_{1}$ & $\neg_{2}$ & \urcorner$_{3}$ & \urcorner$_{4}$ \\
\hline$\lrcorner_{1}$ & $(0.85,0.25 .0 .15)$ & $(0.88,0.23,0.14)$ & $(0.78,0.38,0.18)$ & $(0.83,0.39,0.29)$ \\
\lrcorner$_{2}$ & $(0.94,0.04,0.07)$ & $(0.61,0.19,0.39)$ & $(0.63,0.18,0.35)$ & $(0.56,0.49,0.48)$ \\
\lrcorner$_{3}$ & $(0.73,0.13,0.46)$ & $(0.88,0.39,0.19)$ & $(0.87,0.35,0.18)$ & $(0.81,0.13,0.41)$ \\
\lrcorner$_{4}$ & $(0.82,0.12,0.43)$ & $(0.63,0.21,0.55)$ & $(0.53,0.33,0.47)$ & $(0.51,0.23,0.46)$ \\
$\beth_{5}$ & $(0.61,0.33,0.29)$ & $(0.63,0.41,0.28)$ & $(0.74,0.34,0.14)$ & $(0.65,0.32,0.37)$ \\
\hline
\end{tabular}

TABLe 5: Aggregated SF set information.

\begin{tabular}{lcccc}
\hline & $\neg_{1}$ & $\neg_{2}$ & $\neg_{3}$ & \urcorner$_{4}$ \\
\hline$\lrcorner_{1}$ & $(0.788,0.229,0.319)$ & $(0.785,0.315,0.208)$ & $(0.696,0.402,0.297)$ & $(0.767,0.239,0.371)$ \\
\lrcorner$_{2}$ & $(0.807,0.078,0.279)$ & $(0.674,0.246,0.342)$ & $(0.818,0.160,0.227)$ & $(0.919,0.188,0.097)$ \\
\lrcorner$_{3}$ & $(0.814,0.128,0.223)$ & $(0.893,0.165,0.099)$ & $(0.748,0.284,0.223)$ & $(0.677,0.159,0.393)$ \\
$\beth_{4}$ & $(0.702,0.267,0.271)$ & $(0.533,0.324,0.395)$ & $(0.615,0.424,0.433)$ & $(0.573,0.258,0.421)$ \\
$\beth_{5}$ & $(0.639,0.331,0.271)$ & $(0.644,0.298,0.363)$ & $(0.724,0.365,0.224)$ & $(0.685,0.411,0.296)$ \\
\hline
\end{tabular}

TABLE 6: Yager weighted averaging.

\begin{tabular}{lccr}
\hline & SFYWA & SFYOWA & SFYHWA \\
\hline$\lrcorner_{1}$ & $(0.7620,0.3015,0.3038)$ & $(0.7616,0.3031,0.3025)$ & $(0.7732,0.2710,0.3144)$ \\
\lrcorner$_{2}$ & $(0.8183,0.1775,0.2512)$ & $(0.8187,0.1785,0.2505)$ & $(0.8278,0.1582,0.2388)$ \\
\lrcorner$_{3}$ & $(0.7950,0.1916,0.2541)$ & $(0.7958,0.1918,0.2534)$ & $(0.8039,0.1957,0.2268)$ \\
\lrcorner$_{4}$ & $(0.6163,0.3223,0.3830)$ & $(0.6163,0.3230,0.3830)$ & $(0.6183,0.3392,0.3966)$ \\
\lrcorner$_{5}$ & $(0.6752,0.3529,0.2920)$ & $(0.6761,0.3530,0.2918)$ & $(0.6771,0.3680,0.2828)$ \\
\hline
\end{tabular}

TABLE 7: Yager weighted geometric.

\begin{tabular}{lccr}
\hline & SFYWG & SFYOWG & SFYHWG \\
\hline$I_{1}$ & $(0.7580,0.3015,0.3144)$ & $(0.7575,0.3031,0.3133)$ & $(0.7710,0.2710,0.3236)$ \\
$I_{2}$ & $(0.7928,0.1775,0.2737)$ & $(0.7928,0.1785,0.2735)$ & $(0.8130,0.1582,0.2555)$ \\
$J_{3}$ & $(0.7741,0.1916,0.2918)$ & $(0.7746,0.1918,0.2916)$ & $(0.7900,0.1957,0.2535)$ \\
$I_{4}$ & $(0.6056,0.3223,0.3932)$ & $(0.6056,0.3230,0.3932)$ & $(0.6110,0.3392,0.4053)$ \\
$I_{5}$ & $(0.6720,0.3529,0.3012)$ & $(0.6728,0.3530,0.3011)$ & $(0.6743,0.3680,0.2885)$ \\
\hline
\end{tabular}

TABle 8: Score values.

\begin{tabular}{|c|c|c|c|c|c|}
\hline & $\breve{s c} \breve{c}\left(\beth_{1}\right) c$ & $\breve{s c}\left(I_{2}\right)$ & $\breve{s c}\left(I_{3}\right)$ & $\breve{s c} \breve{c}\left(\beth_{4}\right)$ & $\breve{s} \check{c}\left(I_{5}\right)$ \\
\hline SFYWA & 0.631061 & 0.669998 & 0.656079 & 0.559098 & 0.596208 \\
\hline SFYOWA & 0.631075 & 0.670331 & 0.656609 & 0.559115 & 0.596561 \\
\hline SFYHWA & 0.634867 & 0.677689 & 0.665076 & 0.557001 & 0.598448 \\
\hline SFYWG & 0.627303 & 0.651881 & 0.639113 & 0.553654 & 0.593435 \\
\hline SFYOWG & 0.627262 & 0.651912 & 0.639385 & 0.553665 & 0.593733 \\
\hline SFYHWG & 0.632199 & 0.666162 & 0.653417 & 0.552859 & 0.596481 \\
\hline
\end{tabular}

TABLE 9: Ranking of alternatives.

\begin{tabular}{|c|c|c|}
\hline & Score ranking & Best alternative \\
\hline SFYWA & $\breve{s c} \breve{c}\left(I_{2}\right)>\breve{s} \breve{c}\left(I_{3}\right)>\breve{s} \breve{c}\left(I_{1}\right)>\check{s} \check{c}\left(I_{5}\right)>\check{s} \check{c}\left(I_{4}\right)$ & $I_{2}$ \\
\hline SFYOWA & $\breve{s c}\left(I_{2}\right)>\breve{s} \breve{c}\left(I_{3}\right)>\breve{s} \breve{c}\left(I_{1}\right)>\breve{s} \breve{c}\left(I_{5}\right)>\breve{s} \breve{c}\left(I_{4}\right)$ & $a_{2}$ \\
\hline SFYHWA & $\breve{s} \check{c}\left(I_{2}\right)>\breve{s} \breve{c}\left(I_{3}\right)>\breve{s} \breve{c}\left(I_{1}\right)>\breve{s} \breve{c}\left(I_{5}\right)>\breve{s} \breve{c}\left(I_{4}\right)$ & $I_{2}$ \\
\hline SFYWG & $\breve{s} \breve{c}\left(I_{2}\right)>\breve{s} \breve{c}\left(I_{3}\right)>\breve{s} \breve{c}\left(I_{1}\right)>\breve{s} \breve{c}\left(I_{5}\right)>\breve{s} \breve{c}\left(I_{4}\right)$ & $a_{2}$ \\
\hline SFYOWG & $\breve{s c} \breve{c}\left(I_{2}\right)>\breve{s} \check{c}\left(I_{3}\right)>\breve{s} \check{c}\left(I_{1}\right)>\breve{s} \check{c}\left(I_{5}\right)>\breve{s} \check{c}\left(I_{4}\right)$ & $I_{2}$ \\
\hline SFYHWG & $\breve{s} \breve{c}\left(I_{2}\right)>\breve{s} \breve{c}\left(I_{3}\right)>\breve{s} \breve{c}\left(I_{1}\right)>\breve{s} \breve{c}\left(I_{5}\right)>\breve{s} \breve{c}\left(I_{4}\right)$ & $I_{2}$ \\
\hline
\end{tabular}


TABLE 10: Existing aggregation operators.

\begin{tabular}{lcccr}
\hline & SFSWA [37] & SFSOWA [37] & SFSHWA [37] & L - SFWA [29] \\
\hline$\beth_{1}$ & $(0.76,0.30,0.30)$ & $(0.75,0.30,0.30)$ & $(0.77,0.27,0.31)$ & $(0.91,0.06,0.06)$ \\
\lrcorner$_{2}$ & $(0.80,0.17,0.25)$ & $(0.81,0.17,0.25)$ & $(0.82,0.15,0.24)$ & $(0.95,0.01,0.03)$ \\
\lrcorner$_{3}$ & $(0.78,0.19,0.25)$ & $(0.78,0.19,0.25)$ & $(0.79,0.19,0.22)$ & $(0.94,0.02,0.03)$ \\
$\beth_{4}$ & $(0.60,0.32,0.38)$ & $(0.61,0.32,0.38)$ & $(0.79,0.19,0.22)$ & $(0.70,0.07,0.10)$ \\
$\beth_{5}$ & $(0.67,0.35,0.29)$ & $(0.67,0.35,0.29)$ & $(0.66,0.33,0.29)$ & $(0.82,0.09,0.06)$ \\
\hline
\end{tabular}

TABLE 11: Existing aggregation operators.

\begin{tabular}{lcccr}
\hline & L-SFOWA [29] & L-SFHWA [29] & L-SFWG [29] & L - SFOWG [29] \\
\hline$I_{1}$ & $(0.91,0.06,0.06)$ & $(0.99,0.002,0.004)$ & $(0.91,0.07,0.07)$ & $(0.91,0.07,0.07)$ \\
$a_{2}$ & $(0.96,0.01,0.03)$ & $(0.99,0.0001,0.0003)$ & $(0.92,0.02,0.05)$ & $(0.92,0.02,0.05)$ \\
\lrcorner$_{3}$ & $(0.94,0.02,0.03)$ & $(0.99,0.0003,0.001)$ & $(0.91,0.03,0.06)$ & $(0.91,0.03,0.06)$ \\
$a_{4}$ & $(0.70,0.07,0.10)$ & $(0.81,0.003,0.01)$ & $(0.62,0.08,0.12)$ & $(0.62,0.08,0.12)$ \\
$I_{5}$ & $(0.82,0.09,0.06)$ & $(0.96,0.007,0.002)$ & $(0.81,0.10,0.06)$ & $(0.81,0.10,0.06)$ \\
\hline
\end{tabular}

TABLE 12: Overall ranking of the alternatives.

\begin{tabular}{|c|c|c|}
\hline Existing operators & Ranking & Best alternative \\
\hline SFSWA [37] & $\check{s} \check{c}\left(I_{2}\right)>\check{s} \check{c}\left(I_{3}\right)>\check{s} \check{c}\left(I_{1}\right)>\check{s} \check{c}\left(I_{5}\right)>\check{s} \check{c}\left(I_{4}\right)$ & $I_{2}$ \\
\hline SFSOWA [37] & $\breve{s} \check{c}\left(I_{2}\right)>\breve{s} \check{c}\left(I_{3}\right)>\breve{s} \check{c}\left(I_{1}\right)>\breve{s} \check{c}\left(I_{5}\right)>\breve{s} \check{c}\left(I_{4}\right)$ & $\lambda_{2}$ \\
\hline SFSHWA [37] & $\check{s} \check{c}\left(I_{2}\right)>\check{s} \check{c}\left(I_{3}\right)>\check{s} \check{c}\left(I_{1}\right)>\check{s} \check{c}\left(I_{5}\right)>\check{s} \check{c}\left(I_{4}\right)$ & $\lambda_{2}$ \\
\hline L - SFWA [29] & $\check{s} \check{c}\left(I_{2}\right)>\check{s} \check{c}\left(I_{3}\right)>\check{s} \check{c}\left(I_{1}\right)>\check{s} \check{c}\left(I_{5}\right)>\check{s} \check{c}\left(I_{4}\right)$ & $a_{2}^{2}$ \\
\hline L - SFOWA [29] & $\check{s} \check{c}\left(I_{2}\right)>\breve{s} \check{c}\left(I_{3}\right)>\check{s} \check{c}\left(I_{1}\right)>\check{s} \check{c}\left(I_{5}\right)>\check{s} \check{c}\left(I_{4}\right)$ & $\beth_{2}^{2}$ \\
\hline L - SFHWA [29] & $\check{s} \check{c}\left(I_{2}\right)>\check{s} \check{c}\left(I_{3}\right)>\check{s} \check{c}\left(I_{1}\right)>\check{s} \check{c}\left(I_{5}\right)>\check{s} \check{c}\left(I_{4}\right)$ & $د_{2}$ \\
\hline L - SFWG [29] & $\breve{s} \check{c}\left(I_{2}\right)>\breve{s} \check{c}\left(I_{3}\right)>\breve{s} \check{c}\left(I_{1}\right)>\breve{s} \check{c}\left(I_{5}\right)>\breve{s} \check{c}\left(I_{4}\right)$ & $\lambda_{2}$ \\
\hline L - SFOWG [29] & $\breve{s} \check{c}\left(I_{2}\right)>\breve{s} \check{c}\left(I_{3}\right)>\breve{s} \check{c}\left(I_{1}\right)>\breve{s} \check{c}\left(I_{5}\right)>\breve{s} \check{c}\left(I_{4}\right)$ & $\lambda_{2}$ \\
\hline L - SFHWG [29] & $\breve{s} \check{c}\left(I_{2}\right)>\breve{s} \check{c}\left(I_{3}\right)>\breve{s} \check{c}\left(I_{1}\right)>\breve{s} \check{c}\left(I_{5}\right)>\breve{s} \check{c}\left(I_{4}\right)$ & $\lambda_{2}$ \\
\hline
\end{tabular}

TABLE 13: Overall ranking of the alternatives.

\begin{tabular}{|c|c|c|}
\hline Proposed operators & Ranking & Best alternative \\
\hline SFYWA & $\check{s} \check{s}\left(I_{2}\right)>\breve{s} \check{c}\left(I_{3}\right)>\breve{s} \check{c}\left(I_{1}\right)>\check{s} \check{c}\left(I_{5}\right)>\breve{s} \check{c}\left(I_{4}\right)$ & $I_{2}$ \\
\hline SFYOWA & $\check{s} \check{c}\left(I_{2}\right)>\check{s} \check{c}\left(I_{3}\right)>\check{s} \check{c}\left(I_{1}\right)>\check{s} \check{c}\left(I_{5}\right)>\check{s} \check{c}\left(I_{4}\right)$ & $J_{2}$ \\
\hline SFYHWA & $\check{s} \check{c}\left(I_{2}\right)>\breve{s} \check{c}\left(I_{3}\right)>\breve{s} \check{c}\left(I_{1}\right)>\breve{s} \check{c}\left(I_{5}\right)>\breve{s} \check{c}\left(I_{4}\right)$ & $\lambda_{2}$ \\
\hline SFYWG & $\check{s} \check{c}\left(I_{2}\right)>\check{s} \check{c}\left(I_{3}\right)>\check{s} \check{c}\left(I_{1}\right)>\check{s} \check{c}\left(I_{5}\right)>\check{s} \check{c}\left(I_{4}\right)$ & $I_{2}$ \\
\hline SFYOWG & $\check{s} \check{c}\left(I_{2}\right)>\breve{s} \check{c}\left(I_{3}\right)>\breve{s} \breve{c}\left(I_{1}\right)>\breve{s} \check{c}\left(I_{5}\right)>\breve{s} \check{c}\left(I_{4}\right)$ & $a_{2}$ \\
\hline SFYHWG & $\check{s} \check{c}\left(I_{2}\right)>\check{s} \check{c}\left(I_{3}\right)>\check{s} \check{c}\left(I_{1}\right)>\check{s} \check{c}\left(I_{5}\right)>\check{s} \check{c}\left(I_{4}\right)$ & $\beth_{2}$ \\
\hline
\end{tabular}

Step 7: select the optimal alternative according to the maximum score value as follows in Table 9.

We can conclude from this above computational process that location $\beth_{2}$ is the best for the installation of the wind power plant among others, and therefore it is highly recommended.

\section{Comparison Analysis}

We provide some appropriate examples below to test the potential and efficacy of the established decision-making approach and to compare it with the recent findings.

The use of existing methods and different aggregation operators for computed aggregate information is shown in Tables 10 and 11.

Now, according to their aggregated data, we evaluate the ranking of the alternatives in Tables 12 and 13.

\section{Discussion}

From the outcomes of the proposed operators and the other existing methods, we conclude that ranking lists obtained from both the proposed method and the compared methods are the same. The Yager operators with the spherical fuzzy set environment represent a generalized and novel approach to tackle uncertainty in DM problems. The Yager operators with the spherical fuzzy environment are more flexible and effective to evaluate best alternative in real-word problems.

\section{Conclusion}

Spherical FS, which is a general extension of intuitionistic FS, picture FS, is more capable of dealing with incomplete and inconsistent information. Therefore, it is widely used in various fields. Spherical FS tackles the vagueness and uncertain information in real-world complex problems with 
more flexible and effective way. In addition, the Yager norms have a more generalized framework that works effectively to incorporate complex information. We are motivated by the deficiencies of the existing methods and the beneficial features of the Yager AOs to work towards improving a successful merger with SFNs.

In this study, under the spherical fuzzy model, we modified the multiskilled Yager AOs to integrate the benefits and flexibility of both theories. Later, we explore operational laws of SFN to construct spherical fuzzy AOs that comply with the principles of Yager operations. We have established the SFYWA, SFYOWA, SFYHWA, SFYWG, SFYOWG, and SFYHWG operators to aggregate the SFNs. Some of the main characteristics of the proposed operators have been studied, including idempotency, boundedness, and monotonicity.

The main objective of this study is to present a strategy to address MAGDM that includes spherical fuzzy evaluations based on the proposed operators. The theoretical basis of AOs needs to be carefully considered in preparation for their use in MAGDM. A practical example is provided to demonstrate the implementation of the established strategy for the selection of a suitable location for wind power stations. The comparison analysis of our proposed theory was conducted with the existing operators. The superiority of our proposed operators over the existing DM method has been highlighted. We examined the effect of different parameter values on the results of MAGDM issues. In short, this article creates a tool that has the rich properties of Yager AOs and the SF model's flexibility. We will expand our models to spherical hesitant fuzzy set environments in future research.

\section{Data Availability}

No data were used to support this study.

\section{Ethical Approval}

This article does not contain any studies with human participants or animals performed by any of the authors.

\section{Conflicts of Interest}

The authors declare that they have no conflicts of interest.

\section{References}

[1] L. A. Zadeh, "Fuzzy sets," Information and Control, vol. 8, no. 3, pp. 338-353, 1965.

[2] S. Ashraf, S. Abdullah, and A. O. Almagrabi, "A new emergency response of spherical intelligent fuzzy decision process to diagnose of COVID19," Soft Computing, pp. 1-17, 2020.

[3] O. Barukab, S. Abdullah, S. Ashraf, M. Arif, and S. A. Khan, "A new approach to fuzzy TOPSIS method based on entropy measure under spherical fuzzy information," Entropy, vol. 21, no. 12, p. 1231, 2019.

[4] B. Batool, M. Ahmad, S. Abdullah, S. Ashraf, and R. Chinram, "Entropy based pythagorean probabilistic hesitant fuzzy decision making technique and its application for fog-haze factor Assessment problem," Entropy, vol. 22, no. 3, p. 318, 2020.
[5] M. R. Hashmi and M. Riaz, "A novel approach to censuses process by using Pythagorean m-polar fuzzy Dombi's aggregation operators," Journal of Intelligent \& Fuzzy Systems, vol. 38, no. 2, pp. 1977-1995, 2020.

[6] M. Riaz and M. R. Hashmi, "Linear Diophantine fuzzy set and its applications towards multi-attribute decision-making problems," Journal of Intelligent \& Fuzzy Systems, vol. 37, no. 4, pp. 5417-5439, 2019.

[7] M. Riaz and S. T. Tehrim, "Cubic bipolar fuzzy set with application to multi-criteria group decision making using geometric aggregation operators," Soft Computing, vol. 24, no. 21, pp. 16111-16133, 2020.

[8] Z. Xu and R. R. Yager, "Some geometric aggregation operators based on intuitionistic fuzzy sets," International Journal of General Systems, vol. 35, no. 4, pp. 417-433, 2006.

[9] Z. Xu, "Intuitionistic fuzzy aggregation operators," IEEE Transactions on Fuzzy Systems, vol. 15, no. 6, pp. 1179-1187, 2007.

[10] W. Wang and X. Liu, "Intuitionistic fuzzy information aggregation using Einstein operations," IEEE Transactions on Fuzzy Systems, vol. 20, no. 5, pp. 923-938, 2012.

[11] X. Yu and Z. Xu, "Prioritized intuitionistic fuzzy aggregation operators," Information Fusion, vol. 14, no. 1, pp. 108-116, 2013.

[12] Muneeza, S. Abdullah, and M. Abdullah, "New multicriteria group decision support systems for small hydropower plant locations selection based on intuitionistic cubic fuzzy aggregation information," International Journal of Intelligent Systems, vol. 35, no. 6, pp. 983-1020, 2020.

[13] M. J. Khan, P. Kumam, P. Liu, W. Kumam, and S. Ashraf, “A novel approach to generalized intuitionistic fuzzy soft sets and its application in decision support system," Mathematics, vol. 7, no. 8, p. 742, 2019.

[14] L. H. Son, "Generalized picture distance measure and applications to picture fuzzy clustering," Applied Soft Computing, vol. 46, no. C, pp. 284-295, 2016.

[15] B. C. Cuong, Picture Fuzzy Sets-First Results. Part 1, Seminar Neuro-Fuzzy Systems with Applications, Institute of Mathematics, Hanoi, Vietnam, 2013.

[16] B. C. Cuong and V. Kreinovich, "Picture fuzzy sets," Journal of Computer Science and Cybernetics, vol. 30, no. 4, pp. 409-420, 2014.

[17] G. Wei, "Picture fuzzy aggregation operators and their application to multiple attribute decision making," Journal of Intelligent \& Fuzzy Systems, vol. 33, no. 2, pp. 713-724, 2017.

[18] S. Ashraf, T. Mahmood, S. Abdullah, and Q. Khan, "Different approaches to multi-criteria group decision making problems for picture fuzzy environment," Bulletin of the Brazilian Mathematical Society, New Series, vol. 50, no. 2, pp. 373-397, 2019.

[19] M. Khan, P. Kumam, S. Ashraf, and W. Kumam, "Generalized picture fuzzy soft sets and their application in decision support systems," Symmetry, vol. 11, no. 3, p. 415, 2019.

[20] S. Khan, S. Abdullah, L. Abdullah, and S. Ashraf, "Logarithmic aggregation operators of picture fuzzy numbers for multiattribute decision making problems," Mathematics, vol. 7, no. 7, p. 608, 2019.

[21] M. Qiyas, S. Abdullah, S. Ashraf, and M. Aslam, "Utilizing linguistic picture fuzzy aggregation operators for multipleattribute decision-making problems," International Journal of Fuzzy Systems, vol. 22, no. 1, pp. 310-320, 2020.

[22] S. Ashraf, S. Abdullah, T. Mahmood, and M. Aslam, "Cleaner production evaluation in gold mines using novel distance measure method with cubic picture fuzzy numbers," 
International Journal of Fuzzy Systems, vol. 21, no. 8, pp. 2448-2461, 2019.

[23] M. Qiyas, S. Abdullah, S. Ashraf, and L. Abdullah, "Linguistic picture fuzzy Dombi aggregation operators and their application in multiple attribute group decision making problem," Mathematics, vol. 7, no. 8, p. 764, 2019.

[24] S. Ashraf and S. Abdullah, "Some novel aggregation operators for cubic picture fuzzy information: application in multiattribute decision support problem," Granular Computing, pp. 1-16, 2020.

[25] S. Ashraf, S. Abdullah, T. Mahmood, F. Ghani, and T. Mahmood, "Spherical fuzzy sets and their applications in multi-attribute decision making problems," Journal of Intelligent \& Fuzzy Systems, vol. 36, no. 3, pp. 2829-2844, 2019.

[26] S. Ashraf and S. Abdullah, "Spherical aggregation operators and their application in multiattribute group decision-making," International Journal of Intelligent Systems, vol. 34, no. 3, pp. 493-523, 2019.

[27] S. Ashraf, S. Abdullah, M. Aslam, M. Qiyas, and M. A. Kutbi, "Spherical fuzzy sets and its representation of spherical fuzzy t-norms and t-conorms," Journal of Intelligent \& Fuzzy Systems, vol. 36, no. 6, pp. 6089-6102, 2019.

[28] S. Ashraf, S. Abdullah, and T. Mahmood, "Spherical fuzzy Dombi aggregation operators and their application in group decision making problems," Journal of Ambient Intelligence and Humanized Computing, vol. 11, no. 7, pp. 2731-2749, 2019.

[29] Y. Jin, S. Ashraf, and S. Abdullah, "Spherical fuzzy logarithmic aggregation operators based on entropy and their application in decision support systems," Entropy, vol. 21, no. 7, p. 628, 2019.

[30] H. Jin, S. Ashraf, S. Abdullah, M. Qiyas, M. Bano, and S. Zeng, "Linguistic spherical fuzzy aggregation operators and their applications in multi-attribute decision making problems," Mathematics, vol. 7, no. 5, p. 413, 2019.

[31] S. Ashraf, S. Abdullah, and T. Mahmood, "GRA method based on spherical linguistic fuzzy Choquet integral environment and its application in multi-attribute decision-making problems," Mathematical Sciences, vol. 12, no. 4, pp. 263-275, 2018.

[32] M. Rafiq, S. Ashraf, S. Abdullah, T. Mahmood, and S. Muhammad, "The cosine similarity measures of spherical fuzzy sets and their applications in decision making," Journal of Intelligent \& Fuzzy Systems, vol. 36, no. 6, pp. 6059-6073, 2019.

[33] S. Ashraf, S. Abdullah, and L. Abdullah, "Child development influence environmental factors determined using spherical fuzzy distance measures," Mathematics, vol. 7, no. 8, p. 661, 2019.

[34] S. Zeng, A. Hussain, T. Mahmood, M. Irfan Ali, S. Ashraf, and M. Munir, "Covering-based spherical fuzzy rough set model hybrid with TOPSIS for multi-attribute decision-making," Symmetry, vol. 11, no. 4, p. 547, 2019.

[35] F. K. Gündoğdu and C. Kahraman, "A novel fuzzy TOPSIS method using emerging interval-valued spherical fuzzy sets," Engineering Applications of Artificial Intelligence, vol. 85, pp. 307-323, 2019.

[36] S. Ashraf and S. Abdullah, "Emergency decision support modeling for COVID-19 based on spherical fuzzy information," International Journal of Intelligent Systems, vol. 35, no. 11, pp. 1601-1645, 2020.

[37] S. Ashraf, S. Abdullah, and M. Aslam, "Symmetric sum based aggregation operators for spherical fuzzy information: application in multi-attribute group decision making problem,"
Journal of Intelligent \& Fuzzy Systems, vol. 38, no. 4, pp. 5241-5255, 2020.

[38] F. K. Gundogdu and C. Kahraman, "Extension of WASPAS with spherical fuzzy sets," Informatica, vol. 30, no. 2, pp. 269-292, 2019.

[39] S. A. S. Shishavan, F. Kutlu Gündoğdu, E. Farrokhizadeh, Y. Donyatalab, and C. Kahraman, "Novel similarity measures in spherical fuzzy environment and their applications," Engineering Applications of Artificial Intelligence, vol. 94, p. $103837,2020$.

[40] F. K. Gündoğdu and C. Kahraman, "A novel spherical fuzzy analytic hierarchy process and its renewable energy application," Soft Computing, vol. 24, no. 6, pp. 4607-4621, 2020.

[41] F. K. Gündoğdu and C. Kahraman, "A novel spherical fuzzy QFD method and its application to the linear delta robot technology development," Engineering Applications of Artificial Intelligence, vol. 87, p. 103348, 2020.

[42] M. Akram and G. Shahzadi, "A hybrid decision-making model under q-rung orthopair fuzzy Yager aggregation operators," Granular Computing, pp. 1-15, 2020.

[43] M. Akram, X. Peng, and A. Sattar, "Multi-criteria decisionmaking model using complex pythagorean fuzzy yager aggregation operators," Arabian Journal for Science and Engineering, pp. 1-27, 2020.

[44] G. Shahzadi, M. Akram, and A. N. Al-Kenani, "decisionmaking approach under Pythagorean fuzzy Yager weighted operators," Mathematics, vol. 8, no. 1, p. 70, 2020.

[45] H. Garg, G. Shahzadi, and M. Akram, "Decision-making analysis based on fermatean fuzzy yager aggregation operators with application in COVID-19 testing facility," Mathematical Problems in Engineering, vol. 2020, Article ID 7279027, 16 pages, 2020

[46] K. Attanassov, "Intuitionistic fuzzy sets," Fuzzy Sets and Systems, vol. 20, pp. 87-96, 1986.

[47] R. R. Yager, "Pythagorean membership grades in multicriteria decision making," IEEE Transactions on Fuzzy Systems, vol. 22, no. 4, pp. 958-965, 2013.

[48] T. Mahmood, K. Ullah, Q. Khan, and N. Jan, "An approach toward decision-making and medical diagnosis problems using the concept of spherical fuzzy sets," Neural Computing and Applications, vol. 31, no. 11, pp. 7041-7053, 2019.

[49] R. R. Yager, "Aggregation operators and fuzzy systems modeling," Fuzzy Sets and Systems, vol. 67, no. 2, pp. 129-145, 1994.

[50] T. Höfer, Y. Sunak, H. Siddique, and R. Madlener, "Wind farm siting using a spatial Analytic Hierarchy Process approach: a case study of the Städteregion Aachen," Applied Energy, vol. 163, pp. 222-243, 2016.

[51] D. Latinopoulos and K. Kechagia, "A GIS-based multi-criteria evaluation for wind farm site selection. A regional scale application in Greece," Renewable Energy, vol. 78, pp. 550-560, 2015.

[52] D. Pamučar, L. Gigović, Z. Bajić, and M. Janoš ević, “Location selection for wind farms using GIS multi-criteria hybrid model: an approach based on fuzzy and rough numbers," Sustainability, vol. 9, no. 8, p. 1315, 2017. 